\title{
Store-Collect in the Presence of Continuous Churn with Application to Snapshots and Lattice Agreement ${ }^{\star}$
}

\author{
Hagit Attiya ${ }^{1}$, Sweta Kumari ${ }^{1}$, Archit Somani ${ }^{1}$, and Jennifer L. Welch ${ }^{2}$ \\ ${ }^{1}$ Department of Computer Science, Technion, Israel \\ \{hagit, sweta, archit\}@cs.technion.ac.il \\ ${ }^{2}$ Department of Computer Science and Engineering, Texas A\&M University \\ welchecse.tamu.edu
}

\begin{abstract}
We present an algorithm for implementing a store-collect object in an asynchronous crash-prone message-passing dynamic system, where nodes continually enter and leave. The algorithm is very simple and efficient, requiring just one round trip for a store operation and two for a collect. We then show the versatility of the store-collect object for implementing churn-tolerant versions of useful data structures, while shielding the user from the complications of the underlying churn. In particular, we present elegant and efficient implementations of atomic snapshot and generalized lattice agreement objects that use store-collect.
\end{abstract}

Keywords: Store-collect object · Dynamic message-passing systems · Churn · Crash resilience $\cdot$ Atomic snapshots $\cdot$ Generalized lattice agreement

\section{Introduction}

A popular programming technique that contributes to designing provably-correct distributed applications is to use shared objects for interprocess communication, instead of more low-level techniques. Although shared objects are a convenient abstraction, they are not generally provided in large-scale distributed systems; instead, nodes keep copies of the data and communicate by sending messages to keep the copies consistent.

Dynamic distributed systems allow computing nodes to enter and leave the system at will, either due to failures and recoveries, moving in the real world, or changes to the systems' composition, a process called churn. Motivating applications include those in peer-to-peer, sensor, mobile, and social networks, as well as server farms. We focus on the situation when the network is always fully connected, which could be due to, say, an overlay network. A broadcast mechanism is assumed through which a node can send a message to all nodes present in the system; the broadcast is not necessarily reliable and a message sent by a failing node may not reach some of the nodes.

The usefulness of shared memory programming abstractions has been long established for static systems (e.g., [4, 6]), which have known bounds on the number of fixed computing nodes and the number of possible failures. This success has inspired work on providing the same for newer, dynamic, systems. However, most of this work has

\footnotetext{
* Supported by ISF grant 380/18 and NSF grant 1816922.
} 
shown how to simulate a shared read-write register (e.g., [2, 7, 11, 10, 19]). We discuss a couple of exceptions [12, 22] below.

In this paper, we promote the store-collect shared object [8] (defined in Section 2) as a primitive well-suited for dynamic message-passing systems with an ever-changing set of participants. Each node can store a value in a store-collect object with a STORE operation and can collect the latest value stored by each node with a COLLECT operation. Inherent in the specification of this object is an ability to track the set of participants and to read their latest values.

Below we elaborate on three advantageous features of the store-collect object: The store-collect semantics is well-suited to dynamic systems and can be implemented easily and efficiently in them; the widely-used atomic snapshot object can be implemented on top of a store-collect object; and a variety of other commonly-used objects can be implemented either directly on top of a store-collect or on top of an atomic snapshot object. These implementations are simple and inherit the properties of being churn-tolerant and efficient, showing that store-collect combines algorithmic power and efficiency.

A churn-tolerant store-collect object can be implemented fairly easily. We adopt essentially the same system model as in [7], which allows ongoing churn as long as not too many churn events take place during the length of time that a message is in transit. To capture this constraint, there is an assumed upper bound $D$ on the maximum message delay, but no (positive) lower bound. Nodes do not know $D$ and have no local clocks, causing consensus to be unsolvable [7]. The model differentiates between nodes that crash and nodes that leave; nodes that have entered but not left are considered present even if crashed. The number of nodes that can be crashed at any time is bounded by a fraction of the number of nodes present at that time. During any time interval of length $D$, the number of nodes entering or leaving is a fraction of the number of nodes present in the system at the beginning of the interval. (See Section 3 for model details.)

Our algorithm for implementing a churn-tolerant store-collect object is based on the read-write register algorithm in [7]. It is simple and efficient: once a node joins, it completes a store operation within one round-trip, and a collect operation within two round-trips. The store-collect object satisfies a variant of the "regularity" consistency condition, which is weaker than linearizability [20]. In contrast to our single-round-trip store operation, the write operation in the algorithm of [7] requires two round trips. Another difference between the algorithms is that in ours, each node keeps a local set of tuples with an entry for each known node and its value instead of a single value; when receiving new information, instead of overwriting the single value, our algorithm merges the new information with the old. One contribution of our work in this paper is a significantly revised proof of the churn management protocol that is much simpler than that in [7], consequently making it easier to build on the results. (See Section 4)

Building an atomic snapshot on top of a store-collect object is easy! We present a simple algorithm with an elegant correctness proof (Section 6.2). One may be tempted to implement an atomic snapshot in our model by plugging churn-tolerant registers (e.g., [7]) into the original algorithm of [1]. Besides needlessly sequentializing accesses to the registers, such an implementation would have to track the current set of participants. A store-collect object which encapsulates the changing participants and collects information from them in parallel, yields a simple algorithm very similar in spirit to 
the original but whose round complexity is linear instead of quadratic in the number of participants. The key subtlety of the algorithm is the mechanism for detecting when a scan can be borrowed in spite of difficulties caused by the churn, in order to ensure termination.

Atomic snapshot objects have numerous uses in static systems, e.g., to build multiwriter registers, concurrent timestamp systems, counters, and accumulators, and to solve approximate agreement and randomized consensus (cf. [1, 4]). In addition to analogous applications, we show (Section 6.3) how a churn-tolerant atomic snapshot object can be used to provide a churn-tolerant generalized lattice agreement object [16]. This object supports a PROPOSE operation whose argument is a value belonging to a lattice and whose response is a lattice value that is the join of some subset of all prior input values, including its own argument. Generalized lattice agreement is an extension of (single-shot) lattice agreement, well-studied in the static shared memory model [9]. Generalized lattice agreement has been used to implement many objects [14, 16], including atomic snapshots [9], and conflict-free replicated data types [22, 26, 27], e.g., linearizable abort flags, sets, and max registers [22].

The store-collect object specification is versatile. Our atomic snapshot and generalized lattice agreement algorithms demonstrate that layering linearizability on top of a store-collect object is easy. Yet not every application needs the costs associated with linearizability, and store-collect gives the flexibility to avoid them. Our approach to providing churn-tolerant shared objects is modular, as the underlying complications of the message-passing and the churn is hidden from higher layers by our store-collect implementation. As evidence, we observe (Section 6.1) that store-collect allows very simple implementations of max-registers, abort flags, and sets, in which an implemented operation takes at most a couple of store and collect operations. The choice of problems and the algorithms follow [22] but the algorithms inherit good efficiency and churntolerance properties from our store-collect implementation.

Related work: An algorithm that directly implements an atomic snapshot object in a static message-passing system, bypassing the use of registers, is presented in [15]. This algorithm includes several nice optimizations to improve the message and round complexities. These include speeding up the algorithm by parallelizing the collect, as is already encapsulated in our store-collect algorithm. Our atomic snapshot algorithm works in a dynamic system and has a shorter and simpler proof of linearizability.

Aguilera [3] presents a specification and algorithm for atomic snapshots in a $d y$ namic model in which nodes can continually enter and communicate via shared registers. This algorithm is then used for group membership and mutual exclusion in that model. Variations of the model were proposed in [18, 24], which provided algorithms for election, mutual exclusion, consensus, collect, snapshot, and renaming. Spiegelman and Keidar [25] present atomic snapshot algorithms for a crash-prone dynamic system in which processes communicate via shared registers. Their algorithms uniquely identify each scan operation with a version number to help determine when a scan can be borrowed; we use a similar mechanism in our snapshot algorithm. However, our atomic snapshot algorithm uses a shared store-collect object which tolerates ongoing churn. Our use of a non-linearizable building block requires a more delicate approach to prov- 
ing linearizability, as we cannot simply choose, say, a specific write to an atomic register as the linearization point of an update, as can be done in [25].

The problem of implementing shared objects in the presence of ongoing churn and crash failures in message-passing systems is studied in [10, 11], which considers readwrite registers, and [12], which considers sets. Unlike our results, these papers assume the system size is restricted to a fixed window and the system is eventually synchronous. Like our algorithms, the set algorithm in [12] uses unbounded local memory at the nodes.

A popular alternative way to model churn in message-passing systems is as a sequence of quorum configurations, each of which consists of a set of nodes and a quorum system over that set (e.g., [2, 17, 19, 21, 22]). Explicit reconfiguration operations replace older configurations with newer ones. The assumptions made in [2, 17, 19, 21, 22] are incomparable with those in [7] and in our paper, as the former assume churn eventually stops while the latter assume the churn is bounded.

Most papers on generalized lattice agreement have assumed static systems (cf. [9, 14, 16, 26, 27]. A notable exception is [22], which considers dynamic systems subject to changes in the composition due to reconfiguration. This paper provides an implementation for a large class of shared objects, including conflict-free replicated data types, that can be modeled as a lattice. By showing how to view the state of the system as a lattice as well, the paper elegantly combines the treatment of the reconfiguration and the operations on the object. Unlike our work, the algorithms in [22] require that changes to the system composition eventually cease in order to ensure progress.

\section{The Store-Collect Problem}

A shared store-collect object [8] supports concurrent store and collect operations performed by some set of clients. Each operation has an invocation and response. For a store operation, the invocation is of the form $\operatorname{STORE}_{p}(v)$, where $v$ is a value drawn from some set and $p$ indicates the invoking client, and the response is of the form $\mathrm{ACK}_{p}$, indicating that the operation has completed. For a collect operation, the invocation is of the form COLLECT $p$ and the response is of the form $\operatorname{ReTURN}_{p}(V)$, where $V$ is a view, that is, a set of client-value pairs without repetition of client ids. We use the notation $V(p)$ to indicate $v$ if $\langle p, v\rangle \in V$ and $\perp$ if no pair in $V$ has $p$ as its first element.

Informally, the behavior required of a store-collect object is that each collect operation should return a view containing the latest value stored by each client. We do not require the store and collect operations to appear to occur instantaneously, that is, the object is not necessarily linearizable. Instead, we give a precise definition of the required behavior that is along the lines of interval linearizability [13] or the specification of regular registers [23].

A sequence $\sigma$ of invocations and responses of store and collect operations is a schedule if, for each client id $p$, the restriction of $\sigma$ to invocations and responses by $p$ consists of alternating invocations and matching responses, beginning with an invocation. Each invocation and its matching following response (if present) together make an operation. If the response of operation $o p$ comes before the invocation of operation $o p^{\prime}$ in $\sigma$, then we say op precedes $o p^{\prime}$ (in $\sigma$ ) and $o p^{\prime}$ follows op. We assume that every value writ- 
ten in a store operation in a schedule is unique (a condition that can be achieved using sequence numbers and client ids).

Given two views $V_{1}$ and $V_{2}$ returned by two collect operations in a schedule $\sigma$, we write $V_{1} \preceq V_{2}$ if, for every $\left\langle p, v_{1}\right\rangle \in V_{1}$, there exists $v_{2}$ such that $\left\langle p, v_{2}\right\rangle \in V_{2}$ such that either $v_{1}=v_{2}$ or the $\operatorname{STORE}_{p}\left(v_{1}\right)$ invocation does not occur after the response of $\operatorname{STORE}_{p}\left(v_{2}\right)$ in $\sigma$.

A schedule $\sigma$ satisfies regularity for the store-collect problem if:

- For each collect operation cop in $\sigma$ that returns $V$ and every client $p$, if $V(p)=\perp$, then no store operation by precedes cop in $\sigma$. If $V(p)=v \neq \perp$, then there is a $\operatorname{STORE}_{p}(v)$ invocation that occurs in $\sigma$ before cop completes and no other store operation by $p$ occurs in $\sigma$ between this invocation and the invocation of $c o p$.

- For every two collect operations in $\sigma$, cop $_{1}$ which returns $V_{1}$ and $\operatorname{cop}_{2}$ which returns $V_{2}$, if $\operatorname{cop}_{1}$ precedes $\operatorname{cop}_{2}$ in $\sigma$, then $V_{1} \preceq V_{2}$.

\section{System Model}

We model each node $p$ as a state machine with a set of states, containing two initial states $s_{p}^{i}$ and $s_{p}^{\ell}$. Initial state $s_{p}^{i}$ is used if $p$ is initially in the system, whereas $s_{p}^{\ell}$ is used if $p$ enters the system later. The set of all nodes that are initially in the system is denoted by $S_{0}$. It is finite and nonempty.

State transitions are triggered by the occurrences of events. Possible triggering events are: entering the system $\left(\operatorname{ENTER}_{p}\right)$, leaving the system $\left(\operatorname{LEAVE}_{p}\right)$, receipt of a message $m\left(\operatorname{RECEIVE}_{p}(m)\right)$, invocation of an operation $\left(\operatorname{CoLleCt}_{p}\right.$ or $\left.\operatorname{STORE}_{p}(v)\right)$, and crashing $\left(\mathrm{CRASH}_{p}\right)$.

A step of a node $p$ is a 5-tuple $\left(s^{\prime}, T, m, R, s\right)$ where $s^{\prime}$ is the old state, $T$ is the triggering event, $m$ is the message to be sent, $R$ is a response $\left(\operatorname{RETURN}_{p}(V), \operatorname{ACK}_{p}\right.$, or $\mathrm{JOINED}_{p}$ ) or $\perp$, and $s$ is the new state. The values of $m, R$ and $s$ are determined by a transition function applied to $s^{\prime}$ and $T$. $\operatorname{RETURN}_{p}(V)$ is the response to $\operatorname{CoLLECT}_{p}$, $\mathrm{ACK}_{p}$ is the response to $\mathrm{STORE}_{p}$, and $\mathrm{JOINED}_{p}$ is the response to $\operatorname{ENTER}_{p}$. If $T$ is $\mathrm{CRASH}_{p}$, then $m$ is $\perp$ and $R$ is $\perp$.

A local execution of a node $p$ is a sequence of steps such that:

- the old state of the first step is an initial state;

- the new state of each step equals the old state of the next step;

- if the old state of the first step is $s_{p}^{i}$, then no $\operatorname{ENTER}_{p}$ event occurs;

- if the old state of the first step is $s_{p}^{\ell}$, then the triggering event in the first step is ENTER $_{p}$ and there is no other occurrence of ENTER $p$; and

- at most one of $\mathrm{CRASH}_{p}$ and $\operatorname{LEAVE}_{p}$ occurs and if so, it is in the last step.

In our model, a node that leaves the system cannot re-enter with the same id. It can, however, re-enter with a new id. Likewise, a node that crashes does not recover. A node that crashes and recovers, but loses its state, can re-enter with a new id. Because nodes cannot measure time, a node that crashes and recovers, retaining its state, can be treated as if no crash occurred.

A point in time is represented by a nonnegative real number. A timed local execution is a local execution whose steps occur at nondecreasing times. If a local execution is 
infinite, the times at which its steps occur must increase without bound. Given a timed local execution of a node, if $\left(s^{\prime}, T, m, R, s\right)$ is the step with the largest time less than or equal to $t$, then $s$ is the state of that node at time $t$. A node $p$ is said to be present at time $t$ if it entered the system (i.e., its first step has time at most $t$ ) but has not left (i.e., $\operatorname{LEAVE}_{p}$ does not occur at or before $t$ ). The number of nodes that are present at time $t$ is denoted by $N(t)$. A crashed node (i.e., a node for which $\mathrm{CRASH}_{p}$ occurs at or before $t$ ) is still considered to be present. A node is said to be active at time $t$ if it is present and not crashed at $t$.

An execution $e$ is a possibly infinite set of timed local executions, one for each node that is ever present in the system, such that there is a nonempty finite set of nodes that are initially members. Formally, the first step of each node $p \in S_{0}$ occurs at time 0 and the first step of each other node occurs after time 0 .

We assume a reliable broadcast communication service that provides nodes with a mechanism to send the same message to all nodes 1 in the system; message delivery is FIFO. However, if a broadcast invocation is the last thing that a node does before crashing, the message is not guaranteed to be received by all the nodes; this is a weaker assumption than that made in [7]. If a message $m$ sent at time $t$ is received by a node at time $t^{\prime}$, then the delay of this message is $t^{\prime}-t$. This encompasses transmission delay as well as time for handling the message at both the sender and receiver. Let $D>0$ denote the maximum message delay that can occur in the system. Formally:

- Every sent message has at most one matching receipt at each node and every message receipt has exactly one matching message send.

- If a message $m$ is sent by a node $p$ at time $t, p$ 's next event is not $\mathrm{CRASH}_{p}$, and node $q$ is active throughout $[t, t+D]$ (i.e., $q$ enters by time $t$ and does not leave or crash by time $t+D$ ), then $q$ receives $m$. The delay of every received message is in $(0, D]$.

- Messages from the same sender are received in the order they are sent (i.e., if node $p$ sends message $m_{1}$ before sending message $m_{2}$, then no node receives $m_{2}$ before it receives $m_{1}$ ). This can be achieved by tagging each message with the id of its sender and a sequence number.

Let $\alpha>0$ and $0<\Delta \leq 1$ be real numbers that denote the churn rate and failure fraction, respectively. Let $N_{\min }$ be a positive integer, the minimum system size. The parameters $\alpha$ and $\Delta$ are known to the nodes, but $N_{\min }$ and $D$ are not. We assume executions satisfy three assumptions:

Churn Assumption For all times $t>0$, there are at most $\alpha \cdot N(t)$ ENTER and LEAVE events in $[t, t+D]$.

Minimum System Size For all times $t \geq 0, N(t) \geq N_{\min }$.

Failure Fraction Assumption For all times $t \geq 0$, at most $\Delta \cdot N(t)$ nodes are crashed at time $t$.

A node $p$ is said to be a member at time $t$ if it has joined the system (i.e., $p \in S_{0}$ or $\mathrm{JOINED}_{p}$ occurs at or before $t$ ) but has not left (i.e., $\operatorname{LEAVE}_{p}$ does not occur at or before

\footnotetext{
${ }^{1}$ Sending a message to a single recipient can be accomplished by broadcasting the message and indicating the intended recipient so that others will ignore the message.
} 
$t$ ). Note that, at any time $t$, the members are a subset of the present nodes. It is possible that some members have crashed.

We assume "well-formed" interactions between client threads and their users: An invocation occurs at node $p$ only if $p$ has already joined but has not left or crashed, i.e., $p$ is a member. Furthermore, no previous invocation at $p$ is pending, i.e., at most one operation is pending at each node.

An algorithm is a correct implementation of a store-collect object in our model if the following are true for all executions with well-formed interactions:

- For every node $p \notin S_{0}$, if ENTER $p$ occurs, then at least one of $\operatorname{LEAVE}_{p}, \mathrm{CRASH}_{p}$, or $\mathrm{JOINED}_{p}$ occurs subsequently; that is, every node that enters the system and remains active eventually joins. For every node $p \in S_{0}$, JOINED $p$ never occurs.

- For every node $p$, if $\operatorname{STORE}_{p}(v)$ (respectively, $\operatorname{CoLLECT}_{p}$ ) occurs, then at least one of $\operatorname{LEAVE}_{p}, \mathrm{CRASH}_{p}$, or $\operatorname{ACK}_{p}$ (respectively, $\operatorname{RETURN}_{p}(V)$ ) occurs subsequently; that is, every store or collect operation invoked at a node that remains active eventually completes.

- The schedule resulting from the restriction of the execution to the store and collect invocations and responses satisfies regularity for the store-collect problem.

\section{The Continuous Churn Collect (CCC) Algorithm}

In our algorithm, nodes run client threads, which invoke collect and store operations, and server threads. We assume that the code segment that is executed in response to each event executes without interruption.

To track the composition of the system (Algorithm 11), a node $p$ maintains a set Changes of events concerning the nodes that have entered the system. When an ENTER $p$ event occurs, $p$ adds enter $(p$ ) to its Changes set (Line 1) and broadcasts an enter message requesting information about prior events (Line 2). When $p$ finds out that another node $q$ has entered the system, either by receiving an enter message directly from $q$ or by receiving an enter-echo message for $q$ from a third node, it adds enter $(q)$ to its Changes set (Line 3 or 6). When $p$ receives an enter message from a node $q$, it replies with an enter-echo message containing its Changes set, its current estimate LView (local view) of the state of the simulated object, its flag is_joined indicating whether $p$ has joined yet, and the id $q$ (Line 4). The first time that $p$ receives an enter-echo in response to its own enter message (i.e., one that ends with its own id) from a joined node, it computes join_threshold, the number of enter-echo messages it needs to get before it can join (Line 9) and increments its join_counter (Line 10).

The fraction $\gamma$ is used to calculate join_threshold, the number of enter-echo messages that should be received before joining, based on the size of the Present set (nodes that have entered, but have not left, see Line 97. Setting $\gamma$ is a key challenge in the algorithm as setting it too small might not propagate updated information, whereas setting it too large might not guarantee termination of the join.

When the required number of replies to the enter message sent by $p$ is received (Line 11), $p$ adds join $(q)$ to its Changes set, sets its is_joined flag to true (Line 12), broadcasts a message saying that it has joined (Line 14) and outputs $\mathrm{JOINED}_{p}$ (Line 15b. When $p$ finds out that another node $q$ has joined, either by receiving a join message 
directly from $q$ or by receiving a join-echo message for $q$ from a third node, it adds join $(q)$ to its Changes set (Line 16 or 19). When a LEAVE $p$ event occurs, $p$ broadcasts a leave message (Line 21) and halts (Line 22). When $p$ finds out that another node $q$ is leaving the system, either by receiving a leave message directly from $q$ or by receiving a leave-echo message for $q$ from a third node, it adds leave $(q)$ to its Changes set (Line 23 or 25].

Initially, node $p$ 's Changes set equals $\left\{\operatorname{enter}(q) \mid q \in S_{0}\right\} \cup\left\{\right.$ join $\left.(q) \mid q \in S_{0}\right\}$, if $p \in S_{0}$, and $\emptyset$ otherwise. Node $p$ also maintains a set of nodes that it believes are present: Present $=\{q \mid$ enter $(q) \in$ Changes $\wedge$ leave $(q) \notin$ Changes $\}$, i.e., nodes that have entered, but have not left, as far as $p$ knows. Essentially, Algorithm 1 of CCC is the same as CCREG [7] except for Line 5] which merges newly received information with current local information instead of overwriting it.

Once a node has joined, its client thread can handle collect and store operations (Algorithm 2) and its server thread (Algorithm 3) can respond to clients. The client at node $p$ maintains a derived variable Members $=\{q \mid \operatorname{join}(q) \in \operatorname{Changes} \wedge$ leave $(q) \notin$ Changes $\}$ of nodes that $p$ considers as members, i.e., nodes that have joined but not left.

Our implementation adds a sequence number, sqno, to each value in a view, which is now a set of triples, $\{\langle p, v, s q n o\rangle, \ldots\}$, without repetition of node ids. We use the notation $V(p)=v$ if there exists sqno such that $\langle p, v$, sqno $\rangle \in V$, and $\perp$ if no triple in $V$ has $p$ as its first element.

A merge of two views, $V_{1}$ and $V_{2}$, picks the latest value stored by each node according to the highest sqno. If a triple for node $p$ is in $V_{2}$ but not in $V_{1}$ then the merge includes the triple for $p$ from $V_{2}$ as well, and vice versa. That is,

Definition 1. Given two views $V_{1}$ and $V_{2}$, merge $\left(V_{1}, V_{2}\right)$ is defined as the subset of $V_{1} \cup V_{2}$ consisting of every triple whose node id is in one of $V_{1}$ and $V_{2}$ but not the other, and, for node ids that appear in both $V_{1}$ and $V_{2}$, it contains only the triple with the larger sequence number.

Note that $V_{1}, V_{2} \preceq \operatorname{merge}\left(V_{1}, V_{2}\right)$.

Each node keeps a local copy of the current view in its LView variable. In a collect operation, a client thread requests the latest value of servers' local views using a collectquery message (Line 29). When a server node $p$ receives a collect-query message, it responds with its local view (LView) through a collect-reply message (Line 53) if $p$ has joined the system. When the client receives a collect-reply message, it merges its LView with the received view (RView), to get the latest value corresponding to each node (Line 31). Then the client waits for sufficiently many collect-reply messages before broadcasting the current value of its LView variable in a store message (Line 36). When server $p$ receives a store message with a view RView, it merges $R$ View with its local LView (Line 48) and, if $p$ is joined, it broadcasts store-ack (Line 50). The client waits for sufficiently many store-ack messages before returning LView to complete the collect (Line 47); this threshold is recalculated in Line 34 to reflect possible changes to the system composition that the client has observed.

In a store operation, a client thread updates its local variable LView to reflect the new value by doing a merge (Line 39) and broadcasts a store message (Line 42). When server $p$ receives a store message with view RView, it merges RView with its local 


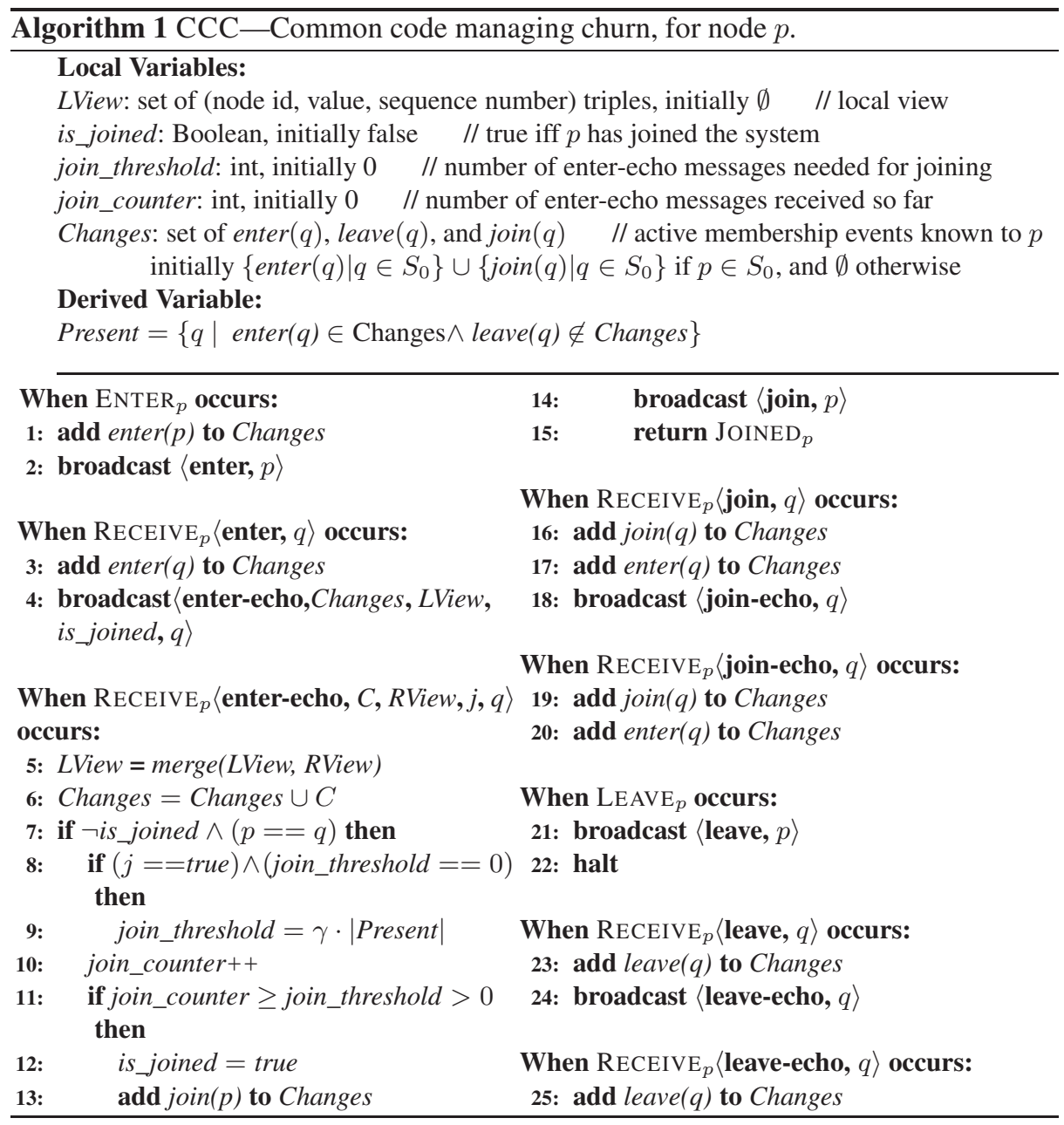

LView (Line 48) and, if $p$ is joined, it broadcasts store-ack (Line 50). The client waits for sufficiently many store-ack messages before completing the store (Line 46).

The fraction $\beta$ is used to calculate the number of messages that should be received (stored in local variable threshold) based on the size of the Members set, for the operation to terminate. Setting $\beta$ is a key challenge in the algorithm as setting it too small might not return correct information from collect or store, whereas setting it too large might not guarantee termination of the collect and store.

We define a phase to be the execution by a client node $p$ of one of the following intervals of its code:

- lines 26 through 33 the first part of a collect operation,

- lines 34 through 36 and 43 through 47 the second part of a collect operation called the "store-back", or

- lines 37 through 46 , the entirety of a store operation. 

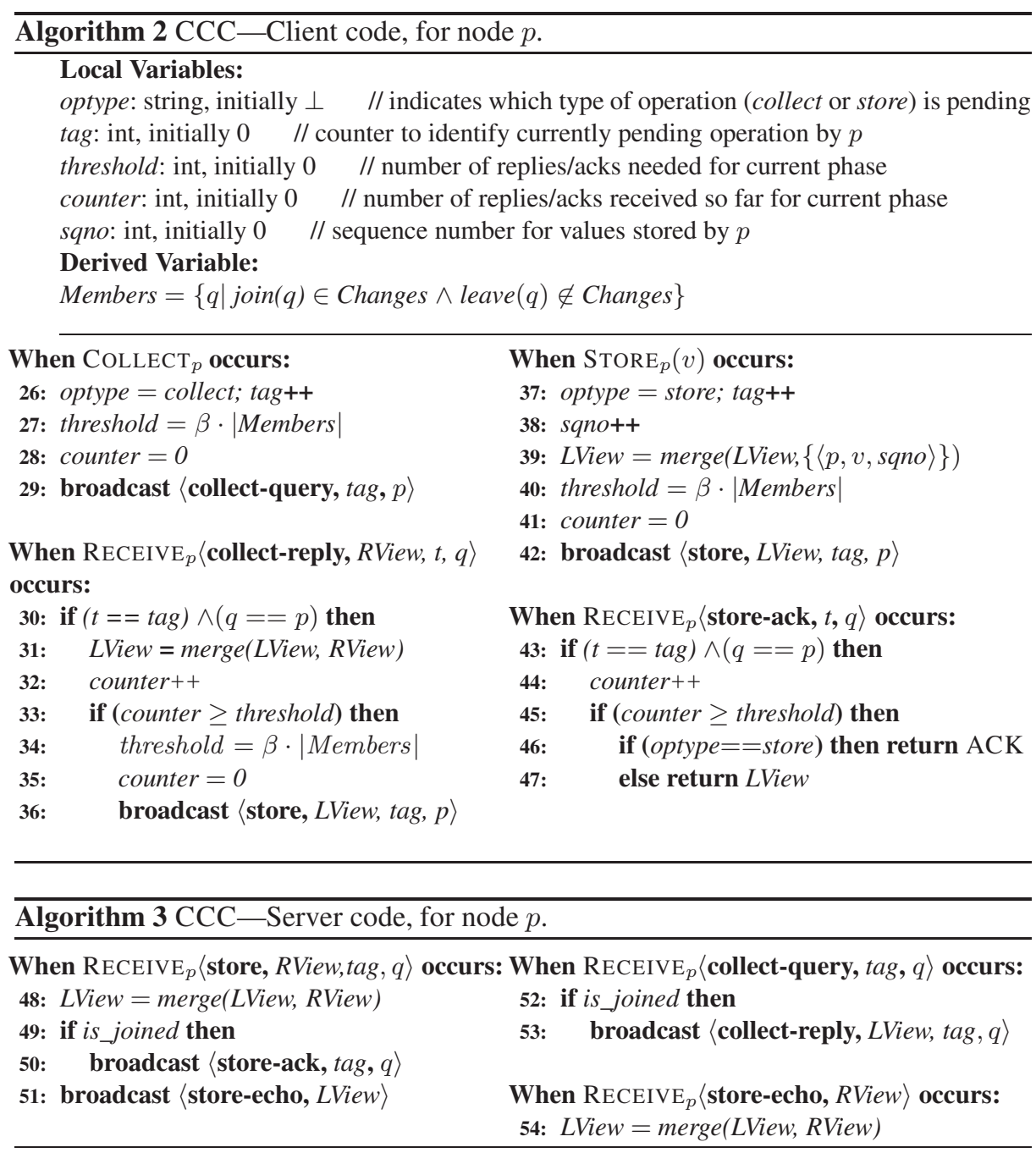

The first kind of phase is called a collect phase while the second and third kinds are called a store phase.

For any completed phase $\varphi$ executed by node $p$, define view $(\varphi)$ to be the value of LView $_{p}^{t}$, where $t$ is the time at the end of the phase. Since a store operation consists solely of a store phase, we also apply the notation to an entire store operation.

\section{Proof of CCC Store-Collect Algorithm}

To prove the correctness of the algorithm, consider any execution of the algorithm. The correctness of the algorithm relies on the following constraints $\left(Z=\left[(1-\alpha)^{3}\right.\right.$ 
$\left.-\Delta \cdot(1+\alpha)^{3}\right]$, which is the fraction of nodes that survive an interval of length $\left.3 D\right)$ :

$$
\begin{aligned}
N_{\min } & \geq \frac{1}{Z+\gamma-(1+\alpha)^{3}} \\
\gamma & \leq Z /(1+\alpha)^{3} \\
\beta & \leq Z /(1+\alpha)^{2} \\
\beta & >\frac{(1-Z)(1+\alpha)^{5}+(1+\alpha)^{6}}{\left((1-\alpha)^{3}-\Delta \cdot(1+\alpha)^{2}\right)\left((1+\alpha)^{2}+1\right)}
\end{aligned}
$$

Fortunately, there are values for the parameters $\alpha, \Delta, \gamma$, and $\beta$ that satisfy these constraints. In the extreme case when $\alpha=0$ (i.e., no churn), the failure fraction $\Delta$ can be as large as 0.21 ; in this case, it suffices to set both $\gamma$ and $\beta$ to 0.79 for any value of $N_{\min }$ that is at least 2 . As $\alpha$ increases up to $0.04, \Delta$ must decrease approximately linearly until reaching 0.01 ; in this case, it suffices to set $\gamma$ to 0.77 and $\beta$ to 0.80 for any value of $N_{\min }$ that is at least 2 . The following technical claims hold:

Lemma 1. For all $i \in \mathbb{N}$ and all $t \geq 0$,

(a) at most $\left((1+\alpha)^{i}-1\right) \cdot N(t)$ nodes enter during $(t, t+i \cdot D]$; and (b) $N(t+i \cdot D) \leq(1+\alpha)^{i} \cdot N(t)$.

Proof. The proof is by induction on $i$.

Basis: $i=0$. For all $t$, the interval $(t, t+0 \cdot D]=(t, t]$ is empty and (a) and (b) are true.

Induction: Assume (a) and (b) are true for $i$ and show for $i+1$. Partition the interval $(t, t+(i+1) \cdot D]$ into $(t, t+D]$ and $(t+D, t+(i+1) \cdot D]$. Since the latter interval is of length $i \cdot D$, the inductive hypothesis applies (replacing $t$ with $t+D$ ) and we get: (a) at most $\left((1+\alpha)^{i}-1\right) \cdot N(t+D)$ nodes enter during $(t+D, t+(i+1) \cdot D]$; and (b) $N(t+(i+1) \cdot D) \leq(1+\alpha)^{i} \cdot N(t+D)$.

By the churn assumption, (i) at most $\alpha \cdot N(t)$ nodes enter during $(t, t+D]$ and thus (ii) $N(t+D) \leq(1+\alpha) \cdot N(t)$. To show (a) for $i+1$, combine (i) with the inductive hypothesis for part (a) to see that the number of nodes that enter during $(t, t+(i+1) \cdot D]$ is

$$
\begin{aligned}
& \leq \alpha \cdot N(t)+\left((1+\alpha)^{i}-1\right) \cdot N(t+D) \\
& \leq \alpha \cdot N(t)+\left((1+\alpha)^{i}-1\right) \cdot(1+\alpha) \cdot N(t) \quad \text { by (ii) } \\
& =\alpha \cdot N(t)+(1+\alpha)^{i+1} \cdot N(t)-(1+\alpha) \cdot N(t) \\
& =\left((1+\alpha)^{i+1}-1\right) \cdot N(t) .
\end{aligned}
$$

To show (b) for $i+1$ :

$$
\begin{aligned}
N(t+(i+1) \cdot D) & \leq(1+\alpha)^{i} \cdot N(t+D) \quad \text { by the inductive hypothesis for (b) } \\
& \leq(1+\alpha)^{i} \cdot(1+\alpha) \cdot N(t) \quad \text { by (ii) } \\
& =(1+\alpha)^{i+1} \cdot N(t) .
\end{aligned}
$$


Calculating the maximum number of nodes that can leave in an interval of length $i \cdot D$ as a function of the number of nodes at the beginning of the interval (i.e., the analog of part (a) of Lemma 1 is somewhat complicated by the possibility of nodes entering during the interval, allowing additional nodes to leave.

Lemma 2. For all $\alpha, 0<\alpha<.206$, all non-negative integers $i \leq 3$, and every time $t \geq 0$, at most $\left(1-(1-\alpha)^{i}\right) \cdot N(t)$ nodes leave during $(t, t+i \cdot D]$.

Proof. The proof is by induction on $i$.

Basis: $i=0$. For all $t$, the interval $(t, t+0 \cdot D]=(t, t]$ is empty and so no nodes leave during it.

Induction: Suppose the lemma is true for $i$ and prove it for $i+1$. Partition the interval $(t, t+(i+1) \cdot D]$ into $(t, t+D]$ and $(t+D, t+(i+1) \cdot D]$. Since the latter interval is of length $i \cdot D$, the inductive hypothesis applies (replacing $t$ with $t+D$ ), stating that the number of nodes that leave in the latter interval is at most $\left(1-(1-\alpha)^{i}\right) \cdot N(t+D)$.

Let $e$ be the exact number of nodes that enter in $(t, t+D]$ and $\ell$ be the exact number of nodes that leave in $(t, t+D]$. The number of nodes that leave in the entire interval is:

$$
\begin{aligned}
& \leq \ell+\left(1-(1-\alpha)^{i}\right) \cdot N(t+D) \quad \text { by the inductive hypothesis } \\
& \leq \ell+\left(1-(1-\alpha)^{i}\right) \cdot[(1+\alpha) \cdot N(t)-2 \ell]
\end{aligned}
$$

The last line is true since $N(t+D)=N(t)+e-\ell$ which equals $N(t)+(\ell+e)-2 \ell$, which is at most $N(t)+\alpha \cdot N(t)-2 \ell$ by the churn assumption. Algebraic manipulations show that this is

$$
\begin{aligned}
& \leq\left(1-(1-\alpha)^{i}\right) \cdot(1+\alpha) \cdot N(t)+\left(2(1-\alpha)^{i}-1\right) \ell \\
& \leq\left(1-(1-\alpha)^{i}\right) \cdot(1+\alpha) \cdot N(t)+\left(2(1-\alpha)^{i}-1\right) \cdot \alpha \cdot N(t)
\end{aligned}
$$

The last line is true since $\ell \leq \alpha \cdot N(t)$ by the churn assumption and $\left(2(1-\alpha)^{i}-1\right)$ is non-negative by the constraints on $\alpha$ and $i$ in the premise of the lemma. This expression equals $\left(1-(1-\alpha)^{i+1}\right) \cdot N(t)$.

Recall that a node is active at time $t$ if it has entered, but not left or crashed, by time $t$. The next lemma counts how many of the nodes that are active at a given time are still active after $3 D$ time has elapsed. It introduces the quantity $Z$ which is the fraction of nodes that must survive an interval of length $3 D$.

Lemma 3. For any interval $\left[t_{1}, t_{2}\right]$ with $t_{2}-t_{1} \leq 3 D$, where $S$ is the set of nodes present at $t_{1}$, at least $Z \cdot|S|$ of the nodes in $S$ are active at $t_{2}$. (Recall that $Z=$ $\left[(1-\alpha)^{3}-\Delta \cdot(1+\alpha)^{3}\right]$.)

Proof. Consider any interval $\left[t_{1}, t_{2}\right]$ with $t_{2}-t_{1} \leq 3 D$ and let $S$ be the set of nodes present at $t_{1}$.

By Lemma 2 at most $\left(1-(1-\alpha)^{3}\right) \cdot|S|$ nodes leave during the interval. In the worst case, all of the leavers are among the original set of nodes $S$. 
By Lemma 1 part (b), the number of nodes present at $t_{2}$ is at most $(1+\alpha)^{3} \cdot|S|$. By the crash assumption, up to a $\Delta$ fraction of them crash, and in the worst case all of these are among the original set of nodes $S$.

Thus the number of nodes in $S$ that remain active at the end of the interval is at least

$$
|S|-\left(1-(1-\alpha)^{3}\right) \cdot|S|-\Delta \cdot(1+\alpha)^{3} \cdot|S|=\left[(1-\alpha)^{3}-\Delta \cdot(1+\alpha)^{3}\right] \cdot|S| .
$$

As an immediate corollary, since $|S|$ must be at least $N_{\text {min }}$, the lower bound on $N_{\text {min }}$ given in Constraint $\mathrm{A}$ shows that at least one node survives. To match its use cases, the corollary is stated with respect to a time that is in the middle of the interval.

Corollary 1 For every $t>0$, at least one node is active throughout the interval $[\max \{0, t-2 D\}, t+D]$.

Throughout the proof, a local variable name is subscripted with $p$ and superscripted with $t$ to denote its value in node $p$ at time $t$; e.g., $v_{p}^{t}$ is the value of node $p$ 's local variable $v$ at time $t$.

In the analysis, we will frequently be comparing the data in nodes' Changes sets to the set of ENTER, JOINED, and LEAVE events that have actually occurred in a certain interval. We refer to these as membership events. We are especially interested in these events that trigger a broadcast invoked by a node that is not in the middle of crashing, as these broadcasts are guaranteed to be received by all nodes that are present for the requisite interval. We call these active membership events. Because of the assumed initialization of the nodes in $S_{0}$, we use the convention that the set of active membership events occurring in the interval $[0,0]$ is $\left\{\operatorname{enter}(p) \mid p \in S_{0}\right\} \cup\left\{\operatorname{join}(p) \mid p \in S_{0}\right\}$.

The next lemmas describe how a node's Changes set relates to prior active membership events. Lemma 4 states that a node that has been present in the system sufficiently long (at least $2 D$ time), has all the information about active membership events up until $D$ time in the past. Lemma 6 states that a joined node, no matter how recently it entered the system, has all the information about active membership events up until $2 D$ time in the past. The later parts of the correctness proof only use Lemma 6 , but its proof relies on Lemma 4. The proof of Lemma 6 relies on Lemma 5, which is rather technical and states that under certain circumstances a node receives an enter-echo message from a long-lived node; we have extracted it as a separate lemma as it is also used later in the proof of Lemma 8 Throughout the proof we denote by $t_{p}^{e}$ the time when event ENTER $_{p}$ occurs.

The proofs of Lemmas 4 and 6 use the next observation, which follows from the fact that nodes broadcast enter/join/leave messages when they enter/join/leave and these messages take at most $D$ time to arrive at active nodes (unless the broadcast is the very last step by a crashing node, in which case the message might not be received by some nodes).

Observation 2 For every node $p$ and all times $t \geq t_{p}^{e}+D$ such that $p$ is active at time $t$, Changes ${ }_{p}^{t}$ contains all the active membership events for $\left[t_{p}^{e}, t-D\right]$. 
Lemma 4. For every node $p$ and all times $t \geq t_{p}^{e}+2 D$ such that $p$ is active at $t$, Changes ${ }_{p}^{t}$ contains all the active membership events for $[0, t-D]$.

Proof. The proof is by induction on the order in which nodes enter. In particular, we consider the nodes in increasing order of ENTER events, breaking ties arbitrarily, and show the properties are true for the current node at all relevant times.

Basis: The first nodes to enter are those in $S_{0}$ and they are assumed to do so at time 0 . Consider $p \in S_{0}$. For $t \geq 2 D$, Observation 2 gives the result.

Induction: Let $p$ be the next node (not in $S_{0}$ ) to enter, at time $t_{p}^{e}$, and assume the lemma is true for all nodes that entered previously.

Consider any time $t \geq t_{p}^{e}+2 D$ such that $p$ is active at $t$. By Corollary 1 there exists a node $q$ that is active throughout $\left[t_{p}^{e}-2 D, t_{p}^{e}+D\right]$. Let $t^{\prime}$ be the time when $q$ receives $p$ 's enter message and $t^{\prime \prime}$ be the time when $p$ receives $q$ 's enter-echo response. We will show that Changes $s_{p}^{t}$ contains all the active membership events for $[0, t-D]$ in three steps: one for $\left[0, t^{\prime}-D\right]$, one for $\left[t^{\prime}-D, t_{p}^{e}\right]$, and one for $\left[t_{p}^{e}, t-D\right]$.

1. Note that $q$ enters the system at least $2 D$ time before it sends its enter-echo message to $p$ at time $t^{\prime}$. By the inductive hypothesis, when $q$ sends that message, its Changes set contains all the active membership events for $\left[0, t^{\prime}-D\right]$. Once $p$ receives the message, at time $t^{\prime \prime}$ which is less than or equal to $t$, its Changes set also contains all the active membership events for $\left[0, t^{\prime}-D\right]$.

2. Suppose some node $r$ enters, joins, or leaves in $\left[t^{\prime}-D, t_{p}^{e}\right]$ and $r$ does not crash during that event. Node $r$ 's enter/join/leave message is received by $q$ either before $t_{p}^{e}$, in which case the information is included in $q$ 's enter-echo message to $p$, or after $t_{p}^{e}$, in which case $q$ sends an enter/join/leave-echo message for $r$, which is received by $p$ before $t$. In either case, the information about $r$ 's event propagates to $p$ before $t$. Thus the result holds for $\left[t^{\prime}-D, t_{p}^{e}\right]$.

3. Observation 2 gives the result for $\left[t_{p}^{e}, t-D\right]$.

Lemma 5. Suppose node $p$ is joined and active at some time $t$ and the first enterecho response that $p$ receives from a joined node $q$ is sent at time $t^{\prime} \leq t$. If Changes $t_{q}^{\prime}$ contains all the active membership events for $\left[0, \max \left\{0, t^{\prime}-2 D\right\}\right]$, then before $p$ joins, it receives an enter-echo response from some node $q^{\prime}$ that is active throughout the interval $\left[\max \left\{0, t^{\prime}-2 D\right\}, t^{\prime}+D\right]$.

Proof. Let $S$ be the set of nodes present at time $\max \left\{0, t^{\prime}-2 D\right\}$. We will show that at least one of the enter-echo responses received by $p$ before joining is from a node in $S$, which is our desired $q^{\prime}$. We start with the value of join_threshold, which is the number of enter-echo responses for which $p$ waits before joining, and then subtract (1) the maximum number of enter-echo responses that could come from nodes not in $S$, (2) the maximum number of nodes in $S$ that could leave too soon (before $t^{\prime}+D$ ), and (3) the maximum number of nodes in $S$ that could crash too soon (before $t^{\prime}+D$ ).

The value of join_threshold is based on the size of $p$ 's Present set at time $t^{\prime \prime}$, immediately after $p$ receives the enter-echo response from $q$ (cf. Line 9 of Algorithm 1). By the premise of the lemma, Changes $t_{q}^{\prime}$ contains all the active membership events for $\left[0, \max \left\{0, t^{\prime}-2 D\right\}\right]$. Thus when $p$ receives the enter-echo response from $q$ at time $t^{\prime \prime} \leq t^{\prime}+D$, its Present variable contains, at a minimum, all the nodes in $S$ minus 
those that left during $\left[\max \left\{0, t^{\prime}-2 D\right\}, t^{\prime \prime}\right]$ —all this quantity $\ell$ —and minus those that crashed while broadcasting their enter message so that $p$ did not receive it — call this quantity $f$. Thus join_threshold $\geq \gamma \cdot(|S|-\ell-f)$.

We now count the maximum number of enter-echo responses that $p$ can receive from nodes not in $S$ before joining. These would be from nodes that enter after $\max \left\{0, t^{\prime}-\right.$ $2 D\}$ but no later than $t^{\prime}+D$, as nodes entering after $t^{\prime}+D$ do not receive $p$ 's enter message. The number of such nodes is $\left((1+\alpha)^{3}-1\right) \cdot|S|$ by Lemma1 part (a).

We then subtract the maximum number of nodes in $S$ that leave before $t^{\prime}+D$. By Lemma2 at most $\left(1-(1-\alpha)^{3}\right) \cdot|S|$ nodes leave during $\left(\max \left\{0, t^{\prime}-2 D\right\}, t^{\prime}+D\right]$. In the worst case, all these leavers are in $S$. Recall that we have already charged $\ell$ to this budget.

Finally we subtract the maximum number of nodes in $S$ that crash before $t^{\prime}+D$. The system size at $t^{\prime}+D$ is at most $(1+\alpha)^{3} \cdot|S|$ by Lemma 1 part (b). At most $\Delta \cdot(1+\alpha)^{3} \cdot|S|$ nodes are crashed at time $t^{\prime}+D$ by the crash assumption. In the worst case, all these crashed nodes are in $S$. Recall that we have already charged $f$ crashes to this budget.

What remains is at least

$$
\begin{aligned}
\gamma \cdot(|S|-\ell-f) & -\left((1+\alpha)^{3}-1\right) \cdot|S| \\
- & {\left[\left(1-(1-\alpha)^{3}\right) \cdot|S|-\ell\right]-\left[\Delta \cdot(1+\alpha)^{3} \cdot|S|-f\right] }
\end{aligned}
$$

which after doing some algebra and using fact that $(1-\gamma)(\ell+f) \geq 0$ is equal to

$$
|S| \cdot\left(\gamma-(1+\alpha)^{3}+(1-\alpha)^{3}-\Delta(1+\alpha)^{3}\right)
$$

Since $|S|$ must be be at least $N_{\min }$, Constraint $\AA$ ensures that the expression is at least one. Thus before $p$ joins, it receives an enter-echo response from at least one node $q^{\prime}$ that is active throughout $\left(\max \left\{0, t^{\prime}-2 D\right\}, t^{\prime}+D\right]$.

Lemma 6. For every node $p$ and all times $t$ such that $p$ is joined and active at $t$, Changes ${ }_{p}^{t}$ contains all the active membership events for $[0, \max \{0, t-2 D\}]$.

Proof. The proof is by induction on the order in which nodes join. In particular, we consider the nodes in increasing order of JoIN events, breaking ties arbitrarily, and show the properties are true for the current node at all relevant times.

Basis: The first nodes to join are those in $S_{0}$ and they are assumed to do so at time 0 . Consider $p \in S_{0}$. When $t \leq 2 D$, we just need to show that Changes ${ }_{p}^{t}$ contains all the active membership events for $[0,0]$, which is true by the assumed initialization of nodes in $S_{0}$. When $t>2 D$, Observation 2 implies the result.

Induction: Let $p$ be the next node (not in $S_{0}$ ) to join and assume the lemma is true for all nodes that previously joined. Consider any time $t$ when $p$ is joined and active.

When $t-t_{p}^{e} \geq 2 D$, Lemma 4 gives the result. So we suppose $t-t_{p}^{e}<2 D$. If $t \leq 2 D$, then all that's required is for Changes ${ }_{p}^{t}$ to include all the active membership events in $[0,0]$. Since $p$ joined, it received an enter-echo message from some previously joined node, which by the inductive hypothesis had all the active membership events for $[0,0]$ in its Changes set when it sent the enter-echo. Thus $p$ receives all the active membership events for $[0,0]$ before it joins. For rest of the proof, assume $t>2 D$. 
We will show that Changes ${ }_{p}^{t}$ contains all the active membership events for $[0, t-2 D]$ in two steps: one for $\left[0, \max \left\{0, t^{\prime}-2 D\right\}\right]$ and one for $\left[\max \left\{0, t^{\prime}-2 D\right\}, t-2 D\right]$ for an appropriately chosen $t^{\prime}<t$.

1. Let $q$ be the first joined node from which $p$ gets an enter-echo response to its enter message. Let $t^{\prime}$ be the time when $q$ sends the enter-echo message. By the inductive hypothesis, since $q$ is joined at $t^{\prime}$, Changes $t_{q}^{t^{\prime}}$ contains all the active membership events for $\left[0, \max \left\{0, t^{\prime}-2 D\right\}\right]$, and thus so does Changes ${ }_{p}^{t}$.

2. By Lemma 5 , receives an enter-echo message at some time before it joins from a node $q^{\prime}$ that is active throughout the interval $\left[\max \left\{0, t^{\prime}-2 D\right\}, t^{\prime}+D\right]$. Let $u^{\prime}$ be the time when $q^{\prime}$ sends its enter-echo response to $p$. Suppose some node $r$ enters, joins or leaves in $\left[\max \left\{0, t^{\prime}-2 D\right\}, t-2 D\right]$ and does not crash during the event. Our goal is to show that $p$ receives the information about $r$ by time $t$. The latest that $r$ 's message is sent is $t-2 D$. Since $t-t^{\prime} \leq t-t_{p}^{e}<2 D$, it follows that $t-D \leq t^{\prime}+D$ and thus $q^{\prime}$ is guaranteed to receive $r$ 's message, as $q^{\prime}$ is still active at $t-D$, the latest that the message could arrive. If $q^{\prime}$ receives $r$ 's message before $u^{\prime}$, then $p$ gets the information about $r$ by time $t$ via the enter-echo response from $q^{\prime}$. Otherwise, $q^{\prime}$ receives $r$ 's message after $u^{\prime}$; the latest this can be is $t-D$. Then $q^{\prime}$ sends an enter-echo message for $r$ which is received by $p$ by time $t$.

We can prove that a node that is active sufficiently long eventually joins.

Theorem 3. Every node $p$ that enters at some time $t$ and is active for at least $2 D$ time joins by time $t+2 D$.

Proof. The proof is by induction on the order in which nodes enter the system.

Basis: The first nodes to enter are those in $S_{0}$ and they are assumed to do so at time 0 . Since they also are assumed to join at time 0 , the theorem follows.

Induction: Let $p$ be the next node (not in $S_{0}$ ) to enter, at time $t_{p}^{e}$, and assume the lemma is true for all nodes that entered previously. Suppose $p$ is active at $t_{p}^{e}+2 D$.

First we show that $p$ receives an enter-echo response to its enter message from at least one joined node.

Suppose $t_{p}^{e}<2 D$. By Corollary 1 , at least one node in $S_{0}$ is active throughout $[0,3 D]$ and thus responds to $p$ 's enter message.

Suppose $t_{p}^{e} \geq 2 D$. By Corollary 1 , there is a node $q$ that is active throughout $\left[t_{p}^{e}-\right.$ $\left.2 D, t_{p}^{e}+D\right]$. Then $q$ enters at least $2 D$ time before $t_{p}^{e}$ and by the inductive hypothesis is joined by $t_{p}^{e}$. Since $q$ is active at least until $t_{p}^{e}+D$, it receives $p$ 's enter message by time $t_{p}^{e}+D$ and sends back an enter-echo which is received by $p$ by time $t_{p}^{e}+2 D$.

We now calculate an upper bound on join_threshold, the number of enter-echo responses for which $p$ waits before joining. This value is based on the size of $p$ 's Present set when it first receives an enter-echo response from a joined node (cf. Line 9 of Algorithm 1). Let $q^{\prime}$ be the sender of this message, let $t^{\prime}$ be the time when the message is sent and $t^{\prime \prime}$ the time when it is received. Since $t^{\prime} \geq t_{p}^{e} \geq 2 D$, it follows that $t^{\prime}-2 D \geq 0$. By Lemma 6, Changes ${ }_{q^{\prime}}^{t^{\prime}}$ contains all the active membership events for $\left[0, t^{\prime}-2 D\right]$ and thus so does Changes $s_{p}^{t^{\prime \prime}}$. As a result, Present $t_{p}^{t^{\prime \prime}}$ contains, at most, all the nodes that are present at time $t^{\prime}-2 D$ (call this set $S$ ) plus the maximum set of nodes that could have 
entered since then. Since $t^{\prime \prime} \leq t^{\prime}+D$, it follows from Lemma 1 part (a) that at most $\left((1+\alpha)^{3}-1\right) \cdot|S|$ nodes enter during $\left(t^{\prime}-2 D, t^{\prime \prime}\right]$. Thus join_threshold $\leq \gamma \cdot(1+\alpha)^{3} \cdot|S|$.

We now show that $p$ is guaranteed to receive at least join_threshold enter-echo responses from nodes in $S$ by time $t_{p}^{e}+2 D$. Each node in $S$ that does not leave or crash by $t_{p}^{e}+D$ receives $p$ 's enter message and sends an enter-echo response by time $t_{p}^{e}+D$, which is received by $p$ by time $t_{p}^{e}+2 D$. The minimum number of such nodes is, by Lemma3 3 and considering the interval $\left(t^{\prime}-2 D, t^{\prime}+D\right]$ :

$$
\begin{aligned}
Z \cdot|S| & \geq \gamma \cdot(1+\alpha)^{3} \cdot|S| \quad \text { by Constraint } \mathbb{B} \\
& \geq \text { join_threshold. }
\end{aligned}
$$

We prove that a phase terminates if the invoking client node is active long enough.

Theorem 4. A phase invoked by a client that remains active completes within $2 D$ time.

Proof. Consider a phase invoked by node $p$ at time $t$. We show that the number of nodes that respond to $p$ 's collect-query or store message is at least as large as the value of threshold computed by $p$ in Line 27 or 34 or 40 of Algorithm 2.

Let $S$ be the set of nodes present at time $\max \{0, t-2 D\}=t^{\prime}$. By Lemma 3 , the number of those nodes that are still active at time $t+D$ is at least $Z \cdot|S|$. If $t^{\prime}=0$, then $S=S_{0}$ and all these nodes are joined throughout; otherwise, by Theorem 3 all these nodes are joined by time $t$.

We now show that $|S| \geq \mid$ Present $t_{p}^{t} \mid /(1+\alpha)^{2}$. By Lemma 6 , Changes ${ }_{p}^{t}$ contains all the active membership events for $\left[0, t^{\prime}\right]$. Present $t_{p}^{t}$ is as large as possible if every node in $S$ succeeds in the broadcast of its enter message, none of the nodes in $S$ leave during $\left[t^{\prime}, t\right]$, and the maximum number of nodes enter during that interval and their enter messages get to $p$ by time $t$. Lemma 1 part (a) implies that the maximum number of nodes that can enter is $(1+\alpha)^{2} \cdot|S|$. Thus $\mid$ Present $_{p}^{t}\left|\leq(1+\alpha)^{2} \cdot\right| S \mid$.

Thus the number of nodes that are joined by time $t$ and are still active at time $t+D$, guaranteed to respond to $p$, is at least

$$
\begin{aligned}
Z \cdot|S| & \geq Z \cdot \mid \text { Present }_{p}^{t} \mid /(1+\alpha)^{2} \\
& \geq \beta \cdot \mid \text { Present }_{p}^{t} \mid \quad \text { by Constraint } \mathbb{C} \\
& \geq \beta \cdot \mid \text { Members }_{p}^{t} \mid
\end{aligned}
$$

The last inequality holds since enter $(q)$ is added to Changes $p$ together with join $(q)$. Since threshold is set to $\beta \cdot \mid$ Members ${ }_{p}^{t} \mid$ at time $t, p$ receives the required number of collect-reply or store-ack messages by time $t+2 D$ and the phase completes.

The following observation is true since in this case node $p$ receives phase $s$ 's store message directly within $D$ time.

Observation 5 For any store phase s that starts at time $t_{s}$ and calls broadcast (Line 42 of Algorithm 21) without crashing during the broadcast, and any node $p$ that is active throughout $\left[t_{s}, t\right]$ where $t \geq t_{s}+D$, view $(s) \preceq$ LView $_{p}^{t}$. 
The next lemma is the analog of Lemma 4 a node that has been active for at least $2 D$ time "knows about" store phases that started up to $D$ in the past.

Lemma 7. If node $p$ is active at any time $t \geq t_{p}^{e}+2 D$, then view $(s) \preceq L$ Liew $w_{p}^{t}$ for every store phase s that starts at or before $t-D$ and calls broadcast (Line 42 of Algorithm 2) without crashing during the broadcast.

Proof. The proof is by induction on the order in which nodes enter the system.

Basis: The first nodes to enter are those in $S_{0}$ and they do so at time 0 . The claim holds by Observation 5

Induction: Let $p$ be the next node (not in $S_{0}$ ) to enter and assume the claim is true for all nodes that entered previously. Consider any time $t \geq t_{p}^{e}+2 D$ when $p$ is active. Let $s$ be a store phase that starts at $t_{s} \leq t-D$ and calls broadcast without crashing. If $t_{s} \geq t_{p}^{e}$, the claim holds by Observation 5

Suppose $t_{s}<t_{p}^{e}$. By Corollary 1 there is at least one node $q$ that is active throughout $\left[\max \left\{0, t_{p}^{e}-2 D\right\}, t_{p}^{e}+D\right]$. Since $t \geq t_{p}^{e}+2 D, p$ receives $q$ 's enter-echo response by time $t$. Since views and sequence numbers are included in enter-echo messages, LView ${ }_{q}^{t^{\prime}} \preceq$ LView $_{p}^{t}$, where $t^{\prime}$ is the time when $q$ receives $p$ 's enter message.

Case 1: $t_{s}<\max \left\{0, t_{p}^{e}-D\right\}$. We show that the inductive hypothesis applies for node $q$, time $t^{\prime}$, and store phase $s$. Thus view $(s) \preceq$ LView $_{q}^{t^{\prime}}$, and by transitivity, $\operatorname{view}(s) \preceq$ LView $_{p}^{t}$. To show that the inductive hypothesis holds, note that $q$ enters before $p, q$ has been active for at least $2 D$ time by $t^{\prime}$ and store phase $s$ starts at or before $t^{\prime}-D$.

Case 2: $t_{s} \geq \max \left\{0, t_{p}^{e}-D\right\}$. The store message sent during $s$ is guaranteed to arrive at $q$ either before $t_{p}^{e}$ or at or after $t_{p}^{e}$. In the former case, $q$ 's enter-echo response, which $p$ receives by $t_{p}^{e}+2 D \leq t$, contains a view $V$ such that view $(s) \preceq V$. In the latter case, $q$ 's store-echo message contains a view $V$ with view $(s) \preceq V$ and $p$ receives this message by $t_{s}+2 D<t_{p}^{e}+2 D \leq t$. In both situations, view $(s) \preceq$ LView $_{p}^{t}$.

The next lemma is the analog of Lemma 6 a node that is joined "knows about" store phases that started up to $2 D$ in the past.

Lemma 8. If node $p$ is joined and active at any time t, then view $(s) \preceq L$ Liew ${ }_{p}^{t}$ for every store phase s that starts at or before $t-2 D$ and calls broadcast (Line 42 of Algorithm [2) without crashing.

Proof. The proof is by induction on the order in which nodes join the system.

Basis: The first nodes to join are those in $S_{0}$ and they do so at time 0 , which is also the time that they enter. The claim holds by Observation 5 .

Induction: Let $p$ be the next node (not in $S_{0}$ ) to join and assume the claim is true for all nodes that joined previously. Consider any time $t$ at which $p$ is joined and active. Let $s$ be any store phase that starts at $t_{s} \leq t-2 D$ and calls broadcast without crashing. If $t \geq t_{p}^{e}+2 D$, then the claim follows from Lemma7

Suppose $t<t_{p}^{e}+2 D$. For every store phase that starts at or after $t_{p}^{e}$, the claim follows from Observation 5

Consider any store phase that starts at some time $t_{s}<t_{p}^{e}$. Let $q$ be the sender of the first enter-echo response received by $p$ from a joined node; suppose the message is sent at $t^{\prime}$ and received at $t^{\prime \prime}$. 
Case 1: $t_{s}<t^{\prime}-2 D$. We show that the inductive hypothesis holds for node $q$, time $t^{\prime}$, and store phase $s$. Thus view $(s) \preceq \operatorname{LView}_{q}^{t^{\prime}}$, and by transitivity, view $(s) \preceq \operatorname{LView}_{p}^{t}$. To show that the inductive hypothesis holds, note that $q$ joins before $p$, it is joined at time $t^{\prime}$, and store phase $s$ starts before $t^{\prime}-2 D$.

Case 2: $t_{s} \geq t^{\prime}-2 D$. Since $q$ is joined at $t^{\prime}$, Lemma 6 implies that Changes $t_{q}^{\prime}$ contains all the active membership events for $\left[0, \max \left\{0, t^{\prime}-2 D\right\}\right]$. Thus Lemma 5 applies and before $p$ joins it receives an enter-echo response from a node $q^{\prime}$ that is active throughout $\left[\max \left\{0, t^{\prime}-2 D\right\}, t^{\prime}+D\right]$. The store message sent during $s$ is guaranteed to arrive at $q^{\prime}$ either before $t_{p}^{e}$ or at or after $t_{p}^{e}$. In the former case, the enter-echo message from $q^{\prime}$ that is sent to $p$ contains a view $V$ with view $(s) \preceq V$; this message is received by $p$ before it joins. In the latter case, the store-echo message from $q^{\prime}$ that is sent to $p$ contains a view $V$ with view $(s) \preceq V$; this message is received by $p$ by $t_{s}+2 D<$ $t_{p}^{e}+2 D \leq t$. In both situations, view $(s) \preceq$ LView $_{p}^{t}$.

The next lemma gives a lower bound on the size of a node's Members set as a function of the size of the system $3 D$ time in the past.

Lemma 9. For every node $p$ and every time $t$ at which $p$ is joined and active, $\mid$ Members $_{p}^{t} \mid \geq\left((1-\alpha)^{3}-\Delta \cdot(1+\alpha)^{2}\right) \cdot N(\max \{0, t-3 D\})$.

Proof. Let $S$ be the set of nodes that are present at time $\max \{0, t-3 D\}$, so $|S|=$ $N(\max \{0, t-3 D\})$.

First, assume $t \geq 3 D$. Since Theorem 3 implies that it takes at most $2 D$ time for a node to join, at a minimum Members ${ }_{p}^{t}$ contains all the nodes in $S$ except for those that leave during $[t-3 D, t]$ and those that crash while broadcasting their join message so that $p$ does not receive the message. By Lemma2, the maximum number of nodes that leave during $[t-3 D, t]$ is $\left(1-(1-\alpha)^{3}\right) \cdot|S|$. To maximize the number of nodes that crash while sending their join message, we consider the largest that the system can be by time $t-D$ (the latest that the nodes in $S$ can join), which is $(1+\alpha)^{2} \cdot|S|$ by Lemma 1 We assume that the maximum number of nodes crash, which is $\Delta \cdot(1+\alpha)^{2} \cdot|S|$, and that all the crashed nodes are in $S$. Thus, $\mid$ Members ${ }_{p}^{t}\left|\geq\left((1-\alpha)^{3}-\Delta \cdot(1+\alpha)^{2}\right) \cdot\right| S \mid$.

Now, assume $t<3 D$. Then $S$ equals $S_{0}$, the set of nodes initially in the system, and Members $_{p}^{t}$ is minimized if no nodes enter and the maximum number of nodes in $S_{0}$ leave and $p$ receives all their leave messages by time $t$. Since Lemma2 implies that the maximum number of nodes that can leave during $[0, t]$ is $\left(1-(1-\alpha)^{3}\right) \cdot|S|$, it follows that $\mid$ Members $_{p}^{t}\left|\geq(1-\alpha)^{3} \cdot\right| S \mid$, which is bigger than the desired lower bound.

To prove the following lemmas, we consider two cases: If the two phases are sufficiently far apart in time, then an information-propagation argument, analogous to that used for the Changes sets, applies. If the two phases are close together in time, then an argument relating to overlapping sets of contacted nodes is used.

Lemma 10. For any store phase $s$ and any collect phase $c$, if $s$ finishes before $c$ starts and c terminates, then view $(s) \preceq$ view $(c)$.

Proof. Let $p_{1}$ be the client node that executes $s$ and $t_{s}$ the start time of $s$. Let $p_{2}$ be the client node that invokes $c$ and $t_{c}$ the start time of $c$. Let $Q_{s}$ be the set of nodes that $p_{1}$ hears from during $s$ (i.e., that sent messages causing $p_{1}$ to increment counter on Line 
44 of Algorithm 2) and $Q_{c}$ be the set of nodes that $p_{2}$ hears from during $c$ (i.e., that sent messages causing $p_{2}$ to increment counter on Line 32 of Algorithm 2).

Case I: $t_{c}-t_{s} \geq 2 D$. Consider any node $q \in Q_{c}$. Since $q$ is in $Q_{c}, q$ is joined when it receives $c$ 's collect-query message at some time, say $t \geq t_{c}$. By the assumption of the case, $t-t_{s} \geq 2 D$. Thus by Lemma 8 , view $(s) \preceq L$ LView ${ }_{q}^{t}$. Since $p_{2}$ receives an enter-echo message from $q$ containing $L$ View ${ }_{q}^{t}$ before completing $c$, it follows that $\operatorname{view}(s) \preceq \operatorname{view}(c)$.

Case II: $t_{c}-t_{s}<2 D$. We will show that $Q_{c}$ and $Q_{s}$ have a nonempty intersection and thus $Q_{c}$ contains a node whose LView variable is $\preceq$ view $(s)$ before it sends its collect-reply message to $p_{2}$, ensuring that $\operatorname{view}(s) \preceq \operatorname{view}(c)$. We define the following sets of nodes.

- Let $J$ be the set of all nodes that are joined and active at some time in $\left[t_{c}, t_{c}+D\right]$.

These are the nodes that could possibly respond to $c$ 's collect-query message. Thus $Q_{c} \subseteq J$.

- Let $K \subseteq Q_{s}$ be the set of nodes in $Q_{s}$ that are still active at $t_{c}$. Note that $K \subseteq J$.

We will show that $\left|Q_{c}\right|+|K|>|J|$. Since $Q_{c}$ and $K$ are both subsets of $J$, it follows that they intersect, and thus $Q_{c}$ and $Q_{s}$ intersect. We show the inequality by calculating an upper bound on $|J|$ and lower bounds on $\left|Q_{c}\right|$ and $|K|$. All three bounds are stated in terms of a common quantity, which is the system size at a particular time $t^{*}=$ $\max \left\{0, t_{c}-2 D\right\}$.

First we calculate an upper bound on $|J|$. Since it takes at most $2 D$ time to join after entering by Theorem 3 , every node in $J$ is either present at $t^{*}$ or enters during $\left[t^{*}, t_{c}+D\right]$. By Lemma1 $\left.\mathrm{b}\right),|J| \leq(1+\alpha)^{3} \cdot N\left(t^{*}\right)$.

Next we calculate a lower bound on $Q_{c}$.

$$
\begin{array}{rlr}
\left|Q_{c}\right| & =\beta \cdot \mid \text { Members }_{p_{2}}^{t_{c}} \mid & \text { by the code } \\
& \geq \beta \cdot\left[(1-\alpha)^{3}-\Delta \cdot(1+\alpha)^{2}\right] \cdot N\left(\max \left\{0, t_{c}-3 D\right\}\right) & \text { by Lemma } 9 \\
& \geq \beta \cdot\left[(1-\alpha)^{3}-\Delta \cdot(1+\alpha)^{2}\right] \cdot(1+\alpha)^{-1} \cdot N\left(t^{*}\right) & \text { by Lemma } 1 \text { (b) }
\end{array}
$$

We now calculate a lower bound on $|K|$. By Lemma 3 , at most $(1-Z) \cdot N\left(t_{s}\right)$ nodes crash or fail during $\left[t_{s}, t_{c}+D\right]$, since the length of the interval is at most $3 D$. In the worst case, all the nodes that crash or fail are in $Q_{s}$.

$$
\begin{aligned}
|K| & \geq\left|Q_{s}\right|-(1-Z) \cdot N\left(t_{s}\right) \\
& =\beta \cdot \mid \text { Members }_{p_{1}}^{t_{s}} \mid-(1-Z) \cdot N\left(t_{s}\right)
\end{aligned}
$$

by the code

$$
\geq \beta \cdot\left[(1-\alpha)^{3}-\Delta \cdot(1+\alpha)^{2}\right] \cdot N\left(\max \left\{0, t_{s}-3 D\right\}\right)-(1-Z) \cdot N\left(t_{s}\right)
$$

by Lemma 9

$$
\geq \beta \cdot\left[(1-\alpha)^{3}-\Delta \cdot(1+\alpha)^{2}\right] \cdot(1+\alpha)^{-3} \cdot N\left(t^{*}\right)-(1-Z) \cdot N\left(t_{s}\right)
$$

by Lemma 1 b) since $0<t_{c}-t_{s}<2 D$

$$
\geq \beta \cdot\left[(1-\alpha)^{3}-\Delta \cdot(1+\alpha)^{2}\right] \cdot(1+\alpha)^{-3} \cdot N\left(t^{*}\right)-(1-Z) \cdot(1+\alpha)^{2} \cdot N\left(t^{*}\right)
$$

by Lemma 1(b) since $0<t_{s}-t^{*}<2 D$ and $1-Z>0$

$$
=\left[\beta \cdot\left[(1-\alpha)^{3}-\Delta \cdot(1+\alpha)^{2}\right] \cdot(1+\alpha)^{-3}-(1-Z) \cdot(1+\alpha)^{2}\right] \cdot N\left(t^{*}\right)
$$


Finally, we show $\left|Q_{c}\right|+|K|>|J|$.

$$
\begin{aligned}
\left|Q_{c}\right|+|K| \geq & {\left[\beta \cdot\left[(1-\alpha)^{3}-\Delta \cdot(1+\alpha)^{2}\right] \cdot(1+\alpha)^{-1}+\beta \cdot\left[(1-\alpha)^{3}\right.\right.} \\
& \left.\left.-\Delta \cdot(1+\alpha)^{2}\right] \cdot(1+\alpha)^{-3}-(1-Z) \cdot(1+\alpha)^{2}\right] \cdot N\left(t^{*}\right) \\
> & (1+\alpha)^{3} \cdot N\left(t^{*}\right) \quad \text { by Constraint } \mathrm{D} \\
\geq & |J| .
\end{aligned}
$$

Theorem 6. The schedule resulting from the restriction of the execution to the store and collect invocations and responses satisfies regularity for the store-collect problem.

Proof. (1) Suppose cop is a collect operation that returns view $V$. Let $c$ be the collect phase of cop. Let $p$ be a node. If $V(p)=\perp$ and a store operation by $p$, consisting of store phase $s$, precedes $c o p$, then, by Lemma 10 , view $(s) \preceq$ view $(c)$. Hence, view $(s)$ contains a tuple for $p$ with a non- $\perp$ value, which is a contradiction.

Therefore, $V(p)=v \neq \perp$. We show that a $\operatorname{STORE}_{p}(v)$ invocation occurs before cop completes and no other store operation by $p$ occurs between this invocation and the invocation of cop. A simple induction shows that every (non- $\perp$ ) value for one node in another node's LView variable at some time comes from a STORE invocation by the first node that has already occurred. Since $V$ is the value of the invoking node's LView variable when cop completes, there is a previous $\operatorname{STORE}_{p}(v)$ invocation.

Now suppose for the sake of contradiction that the $\operatorname{STORE}_{p}(v)$ completes-call this operation sop - and there is another store operation by $p$, call it $s o p^{\prime}$, that follows sop and precedes cop. Let $v^{\prime}$ be the value of $s o p^{\prime}$; by the assumption of unique values, $v \neq v^{\prime}$. Since sop and $s o p^{\prime}$ are executed by the same node, it is easy to see from the code that view $($ sop $) \preceq \operatorname{view}\left(\operatorname{sop}^{\prime}\right)$. By Lemma 10, view $\left(\operatorname{sop}^{\prime}\right) \preceq$ view $(c)=V$. But then value $v$ is superseded by value $v^{\prime} \neq v$, contradicting the assumption that $V(p)=v$.

(2) Suppose $c o p_{1}$ and $c o p_{2}$ are two collect operations such that $c_{0} p_{1}$ returns $V_{1}$, $c o p_{2}$ returns $V_{2}$, and $c o p_{1}$ precedes $c o p_{2}$. Note that $c o p_{1}$ contains a store phase $s$ which finishes before the collect phase $c$ of cop $_{2}$ begins. By Lemma10 view $(s) \preceq$ view $(c)$. Regularity holds since view $(s)=V_{1}$ and view $(c)=V_{2}$, implying that $V_{1} \preceq V_{2}$.

By Theorem 3 , every node that enters and remains active sufficiently long eventually joins. Since a store operation consists of a store phase and a collect operation consists of a collect phase followed by a store phase, by Theorem 4 which states that every phase eventually completes as long as the invoker remains active, every operation eventually completes as long as the invoker remains active. Finally, Theorem 6 ensures regularity.

Corollary $7 \mathrm{CCC}$ is a correct implementation of a store-collect object, in which each Store or Collect completes within a constant number of communication rounds.

\section{Implementing Distributed Objects Despite Continuous Churn}

In this section we show how to implement a variety of objects using store-collect. For all applications, we assume that the conditions for store-collect termination hold, which guarantees termination of the operations. 


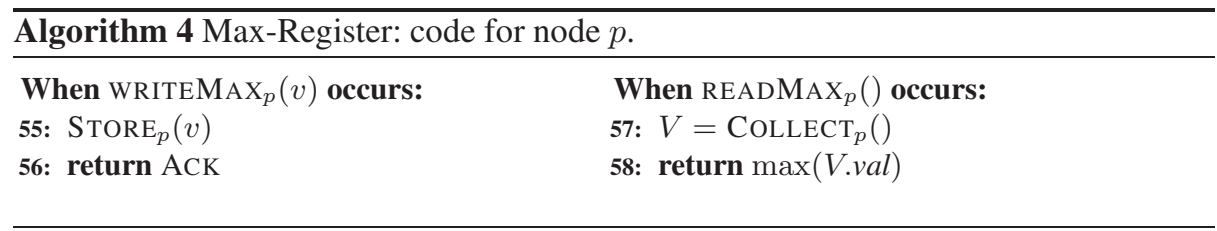

\subsection{Simple, Non-Linearizable Objects}

We start with three simple applications of store-collect for implementing other (nonlinearizable) shared objects 2 The choice of problems and algorithms follow [22], but the algorithms inherit good efficiency properties from our store-collect implementation. Max register: holds the largest value written into it [5]; provides two operations:

- WriteMAX $(v)$ takes a value $v$ as an argument and returns ACK.

- READMAX() has no arguments and returns a value.

Its sequential specification consists of all sequences of WRITEMAX and READMAX operations in which each READMAX returns the largest argument of all preceding WRITEMAX's, if any exists, and 0, if there is none.

Algorithm 44 uses a single store-collect object, holding a single value val for each node, and a local variable $V$ for each node, holding a view. WRITEMAX stores the new value (Line 55) and returns ACK (Line 56). READMAX collects a view (Line 57) and returns the maximum value stored in it (Line 58).

The main correctness properties achieved are as follows. If any value is written before the end of the collect by a READMAX, then by the regularity property of storecollect, the READMAX returns the maximum value of all the values written before it. If the start of the store by a WRITEMAX follows a READMAX, then the READMAX does not consider the store value.

Abort flag: a Boolean flag that can only be raised from false to true [22]; provides two operations:

- ABORT() has no arguments and returns ACK.

- $\operatorname{CHECK}()$ has no arguments and returns true or false.

Its sequential specification consists of all sequences of ABORT and CHECK operations in which each CHECK returns true if an ABORT precedes it, and otherwise returns false.

Algorithm [ follows [22]. It uses a single store-collect object, holding a single flag for each node, and a local variable $F$ for each node, holding a view. ABORT stores true (Line 59) and returns ACK (Line 60). CHECK collects the flags (Line 61). If any of the flags is true then CHECK returns true (Line 62). Otherwise, returns false (Line 63).

\footnotetext{
${ }^{2}$ The behavior of these objects can be formalized through interval linarizability [13].
} 

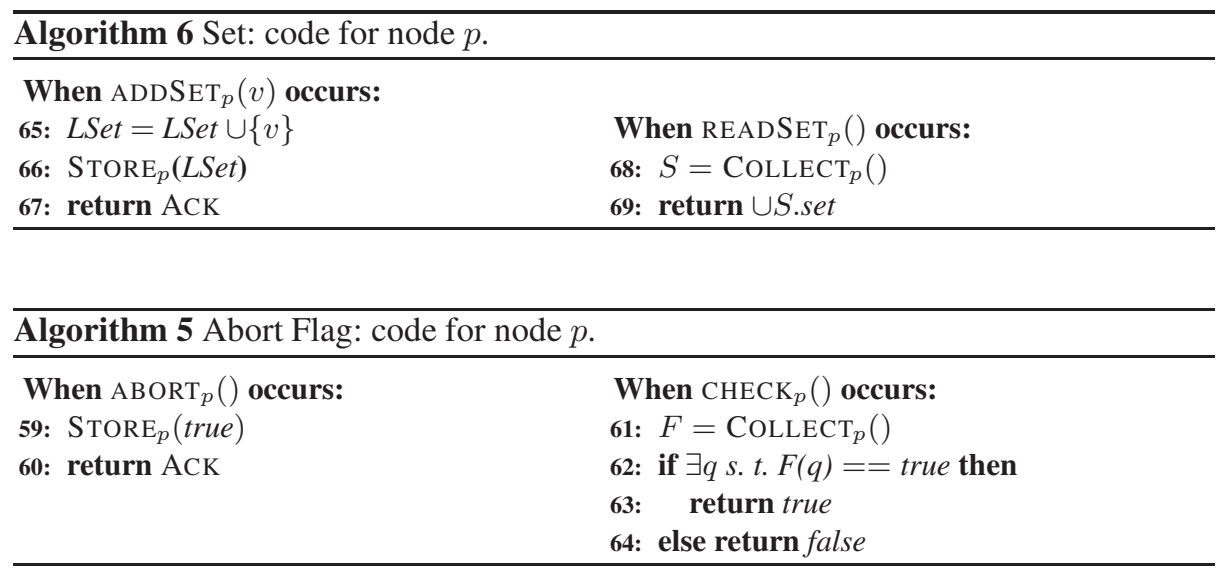

The main correctness property achieved is the following. If an ABORT completes before a CHECK starts, then in particular, its store raises the flag before the end of the collect by CHECK. Hence, the CHECK returns true by the regularity property for storecollect. Otherwise, CHECK returns false.

Set: contains all values added into it [22]; provides two operations:

- $\operatorname{ADDSET}(v)$ takes a value $v$ as an argument and returns ACK.

- READSET() has no arguments and returns a set of values.

Its sequential specification consists of all sequences of ADDSET and READSET operations in which READSET returns the set of all values added by preceding ADDSET operations.

Algorithm 6 uses a store-collect object, holding a set of values for each node, and two local variables for each node: $S$, a view, and $L S e t$, holding all values previously stored by $p$. ADDSET adds the value to the local set (Line 65), stores it (Line 66), and returns ACK (Line 67). READSET collects the set of values (Line 68) and returns the union of all the sets of values (Line 69).

The main correctness property achieved is the following. If an ADDSET stores a value $v$ before the end of a collect by a READSET, then the READSET returns a set of values that includes $v$ by the regularity property for store-collect. Otherwise, the READSET's return value does not include $v$.

\subsection{Atomic Snapshots}

Like other atomic snapshot algorithms [1, 15, 25], our algorithm uses repeated collects to identify an atomic scan when two collects return the same collected views. Updates help scans to complete by embedding an atomic scan that can be borrowed by overlapping scans they interfere with. The set from which the values to be stored in the snapshot object are taken is denoted $\mathrm{Val}_{A S}$. A snapshot view is a subset of $\Pi \times \mathrm{Val}_{A S}$, i.e., a set of (node id, value) pairs, without duplicate node ids.

Formally, an atomic snapshot [1] provides two operations: 
- SCAN(), which has no arguments and returns a snapshot view, and

- $\operatorname{UpDATE}(v)$, which takes a value $v \in V a l_{A S}$ as an argument and returns ACK.

Its sequential specification consists of all sequences of updates and scans in which the snapshot view returned by a SCAN contains the value of the last preceding UPDATE for each node $p$, if such an UPDATE exists, and no value, otherwise.

An implementation should be linearizable [20]. Roughly speaking, for every execution $\alpha$, we should find a sequence of operations, $\operatorname{lin}(\alpha)$, containing all completed operations in $\alpha$ and some of the pending operations, such that:

- $\operatorname{lin}(\alpha)$ is in the sequential specification of an atomic snapshot, and

- $\operatorname{lin}(\alpha)$ preserves the real-time order of non-overlapping operations in $\alpha$.

Our algorithm to implement an atomic snapshot uses a store-collect object, whose values are taken from the set $(\mathcal{P}$ indicates the power set of its argument):

$$
\operatorname{Val}_{S C}=\operatorname{Val}_{A S} \times \mathbb{N} \times \mathbb{N} \times \mathcal{P}\left(\Pi \times V a l_{A S}\right) \times \mathcal{P}(\Pi \times \mathbb{N})
$$

The first component ( $\mathrm{val}$ ) holds the argument of the most recent update invoked at $p$. The second component (usqno) holds the number of updates performed by $p$. The third component (ssqno) holds the number of scans performed by $p$. The fourth component (sview) holds a snapshot view that is the result of a recent scan done by $p$; it is used to help other nodes complete their scans. The fifth component (scounts) holds a set of counts of how many scans have been done by the other nodes, as observed by $p$. The projection of an element $v$ in $\mathrm{Val}_{S C}$ onto a component is denoted, respectively, v.val, v.usqno, v.ssqno, v.sview, v.scounts.

A store-collect view is a subset of $\Pi \times V l_{S C}$, i.e., a set of (node id, value) pairs, with no duplicate node ids. We extend the projection notation to a store-collect view $V$, so that V.comp is the result of replacing each tuple $\langle p, v\rangle$ in $V$ with $\langle p, v$.comp $\rangle$. Recall that for any kind of view $V, V(p)$ is the second component of the pair whose first component is $p$ ( $\perp$ if there is no such pair). Sometimes we restrict attention to those tuples in a view $V$ whose val component is a "real" value, reflecting a store; to this end we use the notation

$$
r(V)=\{\langle p, v\rangle \mid\langle p, v\rangle \in V \text { and } v \cdot v a l \neq \perp\} .
$$

To execute a SCAN, Algorithm 7 increments the scan sequence number (ssqno) (Line 70) and stores it in the shared store-collect object with all the other components unchanged, indicated by the - notation. Then, a view is collected (Line 72). In a while loop, the last collected view is saved and a new view is collected (Line 74). If the two most recently collected views reflect the same set of updates (Line 75), the latest collected view is returned (Line 76); Lines 75 and 76 consider only tuples with "real" values. We call this a successful double collect, and say that this is a direct scan. Otherwise, the algorithm checks whether the last collected view contains a node $q$ that has observed its own ssqno, by checking the scounts component (Line 77). If this condition holds, the snapshot view of $q$ is returned (Line 78); we call this a borrowed scan.

An UPDATE first obtains all scan sequence numbers from a collected view and assigns them to a local variable scounts (Line 79). Next, the value of an embedded scan 


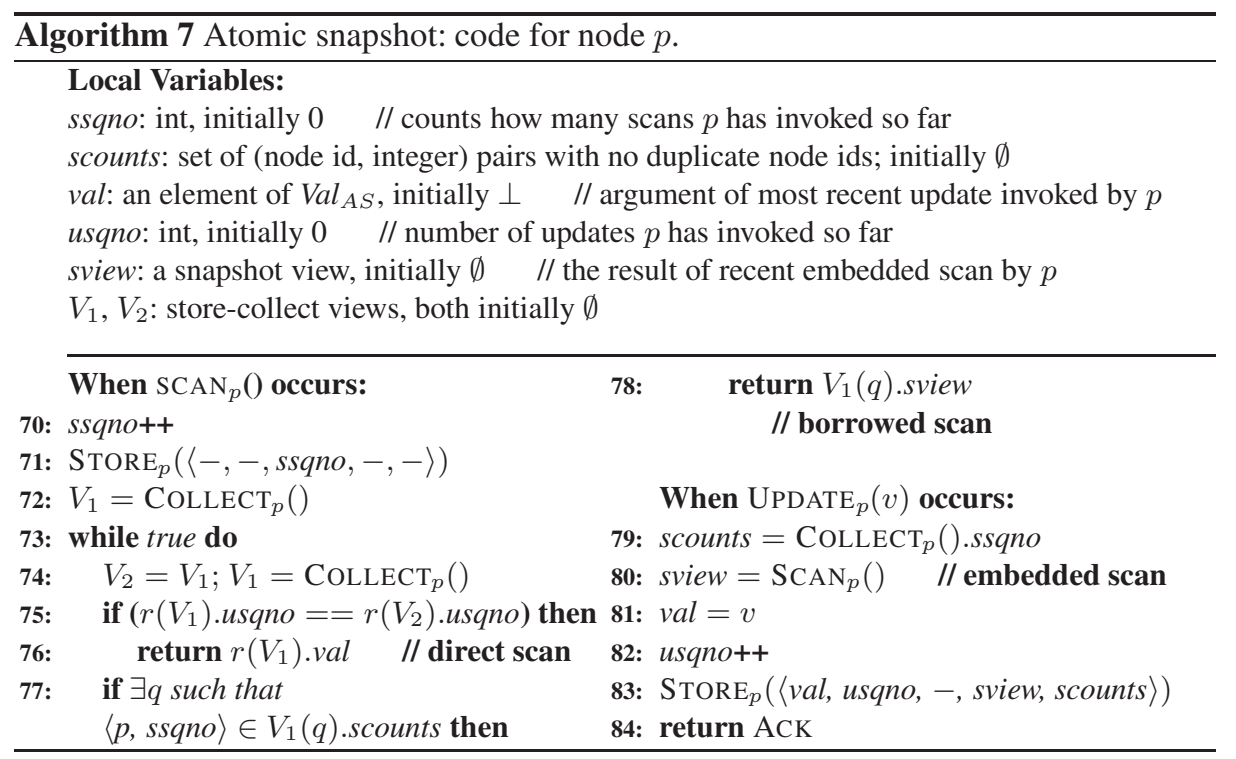

is saved in a local variable sview (Line 80). Then it sets its val variable to the argument value and increments its update sequence number (Lines 81 and 82). Finally the new value, update sequence number, collected view, and set of scan sequence numbers are stored; the node's own scan sequence number is unchanged (Line 83).

To prove linearizability, we consider an execution and specify an ordering of all the completed scans and all the updates whose store on Line 83 started. The ordering takes into consideration the embedded scans, which are inside updates, as well as the "freestanding" scans; since scans do not change the state of the atomic snapshot object, it is permissible to do so.

We first show that the snapshot views returned by direct scans are comparable in the following order: Let $W_{1}$ be the snapshot view returned by a direct scan based on the collect view $V_{1}$ (cf. Line 76) and $W_{2}$ be the snapshot view returned by a direct scan based on the collect view $V_{2}$ (cf. Line 76). We define $W_{1} \preceq W_{2}$ if for every $\langle p, v\rangle \in W_{1}$, there exists $\left\langle p, v^{\prime}\right\rangle \in W_{2}$ where the usqno associated with $v$ in $V_{1}$ is less than or equal to the usqno associated with $v^{\prime}$ in $V_{2}$.

Lemma 11. If a direct scan by node $p$ returns $W_{1}$ and a direct scan by node $q$ returns $W_{2}$, then either $W_{1} \preceq W_{2}$ or $W_{2} \preceq W_{1}$.

Proof. Let $\operatorname{cop}_{p}^{1}$, returning $V_{1}^{\prime}$, followed by $\operatorname{cop}_{p}^{2}$, returning $V_{1}$, be the successful double collect at the end of $p$ 's direct scan and let $c o p_{q}^{1}$, returning $V_{2}^{\prime}$, followed by $c o p_{q}^{2}$, returning $V_{2}$, be the successful double collect at the end of $q$ 's direct scan. Note that $W_{1}=$ $r\left(V_{1}\right) \cdot v a l$, which is equal to $r\left(V_{1}^{\prime}\right) \cdot v a l$, and similarly $W_{2}=r\left(V_{2}\right) \cdot v a l=r\left(V_{2}^{\prime}\right) \cdot v a l$.

Case 1: $\operatorname{cop}_{p}^{1}$ completes before $\operatorname{cop}_{q}^{2}$ starts. Consider any $\langle r, w\rangle \in W_{2}$. Then $\langle r, v\rangle$ is in both $V_{1}^{\prime}$ and $V_{1}$, with $v \cdot v a l=w$ and $v$.usqno $>0$. By regularity of store-collect, $V_{1}^{\prime} \preceq V_{2}$. Thus there is an entry $\left\langle r, v^{\prime}\right\rangle \in V_{2}$ such that either $v=v^{\prime}$ or $v^{\prime}$ is stored by $r$ after $v$ is stored by $r$. Since the usqno variable at $r$ takes on increasing values, v.usqno 
$\leq v^{\prime}$.usqno. Thus there is an entry $\left\langle r, w^{\prime}\right\rangle \in W_{2}$ where the usqno associated with $w^{\prime}$ is at least as large as that associated with $w$. Hence $W_{1} \preceq W_{2}$.

Case 2: $\operatorname{cop}_{q}^{1}$ completes before $\operatorname{cop}_{p}^{2}$ starts. An analogous argument shows that $W_{2} \preceq W_{1}$.

Consider all direct scans in the order they complete and place them by the comparability order. Suppose a direct scan returning snapshot view $W_{1}$, obtained from collect view $V_{1}$, precedes another direct scan returning snapshot view $W_{2}$, obtained from collect view $V_{2}$. The regularity of store-collect ensures that $V_{1} \preceq V_{2}$, and thus $W_{1} \preceq W_{2}$. Hence, this ordering preserves the real-time order of non-overlapping direct scans.

The next lemma helps to order borrowed scans. Its statement is based on the observation that if a scan $s o p_{p}$ by node $p$ borrows the snapshot view in $V_{1}(q)$, then there is an update $u о p_{q}$ by $q$ that writes this view (via a store).

Lemma 12. If a scan $\operatorname{sop}_{p}$ by node $p$ borrows from a scan $\operatorname{sop}_{q}$ by node $q$, then $\operatorname{sop}_{q}$ starts after sop $_{p}$ starts and completes before sop $p_{p}$ completes.

Proof. Let $u o p_{q}$ be the update in which $s o p_{q}$ is embedded. Since $s o p_{p}$ borrows the snapshot view of $s_{0} p_{q}$, its ssqno appears in scounts of $q$ 's value in the view collected in Line 74 The properties of store-collect imply that the collect of $u o p_{q}$ (Line 79) does not complete before the store of $p$ (Line 71) starts. Hence, $\operatorname{sop}_{q}$ (called in Line 80) starts after $\operatorname{sop}_{p}$ starts. Furthermore, since the collect of $p$ returns the snapshot view stored after $s o p_{q}$ completes (Line 83), $s o p_{q}$ completes before $s o p_{p}$ completes.

For every borrowed scan $s o p_{1}$, there exists a chain of scans $\operatorname{sop}_{2}, \operatorname{sop}_{3}, \ldots, \operatorname{sop}_{k}$ such that $\operatorname{sop}_{i}$ borrows from $\operatorname{sop}_{i+1}, 1 \leq i<k$, and $\operatorname{sop}_{k}$ is a direct scan from which $s o p_{1}$ borrows. Consider all borrowed scans in the order they complete and place each borrowed scan after the direct scan it borrows from, as well as all previously linearized borrowed scans that borrow from the same direct scan. Applying Lemma 12 inductively, $\operatorname{sop}_{k}$ starts after $s o p_{1}$ starts and completes before $\operatorname{sop}_{1}$ completes, i.e., the direct scan from which a scan borrows is completely contained, in the execution, within the borrowing scan. This fact, together with the rule for ordering borrowed scans, implies that the real-time order of any two scans, at least one of which is borrowed, is preserved since direct scans have already been shown to be ordered properly.

Finally, we consider all updates in the order their stores (Line 83) start. Place each update, say uop by node $p$ with argument $v$, immediately before the first scan whose returned view includes $\left\langle p, v^{\prime}\right\rangle$, where either $v^{\prime}=v$ or $v^{\prime}$ is the argument of an update by $p$ that follows иор. If there is no such scan, then place uоp at the end of the ordering. Note that all later scans return snapshot views that include $\left\langle p, v^{\prime}\right\rangle$, where either $v^{\prime}=v$ or $v^{\prime}$ is the argument of an update by $p$ that follows uop. This rule for placing updates ensures that the ordering satisfies the sequential specification of atomic snapshots.

Note that if a scan completes before an update starts, then the scan's returned view cannot include the update's value; similarly, if an update completes before a scan starts, then the scan's returned view must includes the update's value or a later one. This shows that the ordering respects the real-time order between non-overlapping updates and scans. The next lemma deals with non-overlapping updates. 
Lemma 13. Let $V$ be the snapshot view returned by a scan sop. If $V(p)$ is the value of an update uop $p_{p}$ by node $p$ and an update uop ${ }_{q}$ by node $q$ precedes uop ${ }_{p}$, then $V(q)$ is the value of uop ${ }_{q}$ or a later update by $q$.

Proof. Let sop' be sop if sop is a direct scan and otherwise the direct scan from which sop borrows. Let $W$ be the (store-collect) view returned by the last two collects, $c o p_{1}$ and $\mathrm{cop}_{2}$, of $\mathrm{sop}^{\prime}$.

We now show that $V=W . v a l$. If $s o p^{\prime}=\operatorname{sop}$, then $V=W$.val by Line 76, since sop is a direct scan. Otherwise, $V=W$.val because $W . v a l$ is returned to the enclosing scan, assigned to sview, and then stored (cf. Lines 80 and 83). This snapshot view is then propagated through the chain of borrowed-from scans and their enclosing updates until reaching sop where it is returned as $V$.

Since $V$ includes the value of $u o p_{p}$, so does $W$. It follows that both stores of $u o p_{p}$ start before $c o p_{1}$ completes and thus before $c o p_{2}$ starts. Since $u o p_{q}$ precedes $u o p_{p}$, the store of $u o p_{q}$ at Line 83 completes before either store of $u o p_{p}$ starts. Thus the store of $u o p_{q}$ completes before $\mathrm{cop}_{2}$ starts, and by the store-collect property, the view $W$ returned by $\operatorname{cop}_{2}$ must include the value of $u o p_{q}$ or a later update by $q$. Since $V=$ $W$.val, the same is true for $V$.

Consider an update $u o p_{p}$, by node $p$, that follows an update $u o p_{q}$, by node $q$, in the execution. If $u o p_{p}$ is placed at the end of the (current) ordering because there is no scan that observes its value or a later update by $p$, then it is ordered after $u o p_{q}$. If $u o p_{p}$ is placed before a scan, then the same must be true of $u o p_{q}$. By construction, the next scan after $u o p_{p}$ in the ordering, call it sop, returns view $V$ with $V(p)$ equal to the value of $u o p_{p}$ or a later update by $p$. By Lemma 13. $V(q)$ must equal the value of $u o p_{q}$ or a later update by $q$. Thus $u o p_{q}$ cannot be placed after sop, and thus it is placed before $u o p_{p}$.

We now consider the termination property of the algorithm. Suppose scan $s o p_{q}$ by node $q$ contains two consecutive collects $c o p_{1}$, which returns $V_{1}$, followed by $c o p_{2}$, which returns $V_{2}$, and this double collect is unsuccessful. Then $V_{1}(p)$.usqno is not equal to $V_{2}(p)$.usqno for some node $p$ and $V_{2}$ does not contain an scounts that includes $s_{0} p_{q}$ 's scan sequence number ssqno. Thus, there exists an update $u о p_{p}$ by node $p$ that is observed by $V_{2}$ but not by $V_{1}$. The correctness of store-collect implies that $u о p_{p}$ finishes after $V_{1}$ starts. Yet $u о p_{p}$ does not include $s o p_{q}$ 's $s s q n o$, which means that $u o p_{p}$ starts before the store at the beginning of $\operatorname{sop}_{q}$ completes. Let $t$ be the time when the store at the beginning of $\operatorname{sop}_{q}$ completes and recall that $N(t)$ denotes the number of nodes present at time $t$. Thus at most $N(t)$ updates are pending at time $t$, implying that $\operatorname{sop}_{q}$ has at most $N(t)$ unsuccessful double collects before it can borrow a scan view. Hence, UPDATE executes at most $O(N(t))$ collects and stores. Putting the pieces together, we have:

Theorem 8. Algorithm 7 is a linearizable implementation of an atomic snapshot object. The number of communication rounds in a SCAN or an UPDATE operation is at most linear in the number of nodes present in the system when the operation starts.

\subsection{Generalized Lattice Agreement}

Let $\langle L, \sqsubseteq\rangle$ be a lattice, where $L$ is the domain of lattice values, ordered by $\sqsubseteq$. We assume a join operator, $\sqcup$, that merges lattice values. A node $p$ calls a PROPOSE operation 


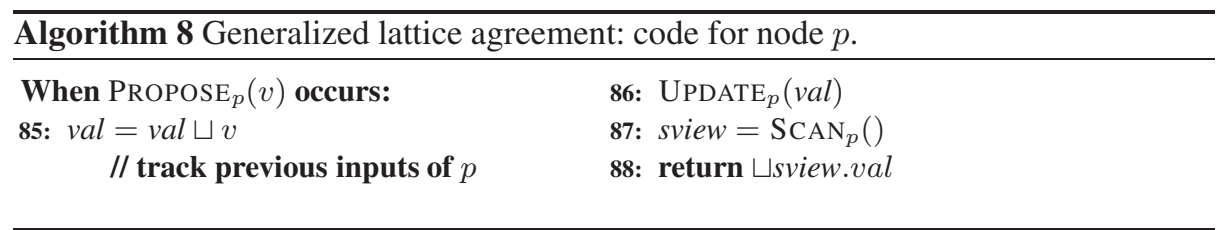

with a lattice input value, and gets back a lattice output value. The input to $p$ 's $i$-th PROPOSE is denoted $v_{i}^{p}$ and the response is $w_{i}^{p}$. The following conditions are required:

Validity Every response value $w_{i}^{p}$ is the join of some values proposed before this response, including $v_{i}^{p}$, and all values returned to any node before the invocation of $p$ 's $i$-th PROPOSE.

Consistency Any two values $w_{i}^{p}$ and $w_{j}^{q}$ are comparable.

This definition is a direct extension of one-shot lattice agreement [9], following [22]. The version studied in [16] is weaker and lacks real-time guarantees across nodes.

Algorithm 8 uses an atomic snapshot object, in which each node stores a single lattice value ( $\mathrm{val}$ ). A PROPOSE operation is simply an UPDATE of a lattice value which is the join of all the node's previous inputs, followed by a SCAN returning the analogous values for all nodes, whose join is the output of PROPOSE.

Validity and consistency are immediate from atomic snapshot properties. Clearly, the algorithm terminates within $O(N)$ collects and stores, where $N$ is the maximum number of nodes concurrently active during the execution of PROPOSE. Since PROPOSE includes one UPDATE and one SCAN, it terminates if the node does not crash or leave.

\section{Conclusion}

We have advocated for the usefulness of the store-collect object as a powerful, flexible, and efficient primitive for implementing a variety of shared objects in dynamic systems with continuous churn. We presented a simple churn-tolerant implementation of storecollect in which the store operation completes within one round trip and the collect operation completes within two. We presented an algorithm for atomic snapshots and another one for generalized lattice agreement using atomic snapshot. The good performance of the underlying store-collect carries over to the latter two problems, since the values can be collected in parallel rather than in series. We also described some simple implementations of non-linearizable objects (max register, abort flag, and set) using store-collect. This assortment of applications highlights the ability to choose whether we want to pay the price of linearizability or settle for the weaker "regularity" condition of store-collect.

If the level of churn is too great, our store-collect algorithm is not guaranteed to preserve the safety property; that is, a collect might miss the value written by a previous store, essentially by the same counter-example as that given in [7]. This behavior is in contrast to the algorithms in [2, 19, 22], which never violate the safety property but only ensure progress once reconfigurations cease. In future work, we would like 
to either improve our algorithm to avoid this behavior or prove that any algorithm that tolerates ongoing churn is subject to such bad behavior.

Our correctness proof for our store-collect algorithm requires that the parameters defining the churn rate and failure fraction satisfy certain conditions. These conditions imply that even in the absence of churn the failure fraction tolerable by our algorithm is smaller than in the static case (namely, less than one-third versus less than one-half). Some degradation is unavoidable when allowing for the possibility of churn, since an argument from [7] can be adapted to show that when implementing store-collect in a system with churn rate $\alpha$, the fraction of failures must be less than $1 /(\alpha+2)$. It would be nice to find less restrictive constraints on the parameters, either through a better analysis or a modified algorithm, or to show that they are necessary.

Another desirable modification to the store-collect algorithm would be reducing the size of the messages and the amount of local storage by garbage-collecting the Changes sets. In the same vein, we would like to know if modifying the atomic snapshot specification to remove from returned views entries of nodes that have left, as is done in [25], can lead to a more space-efficient algorithm.

\section{Acknowledgment}

We thank Luis Pantin for helpful comments.

\section{References}

1. Afek, Y., Attiya, H., Dolev, D., Gafni, E., Merritt, M., Shavit, N.: Atomic snapshots of shared memory. J. ACM 40(4), 873-890 (1993)

2. Aguilera, M.K., Keidar, I., Malkhi, D., Shraer, A.: Dynamic atomic storage without consensus. J. ACM 58(2), 7:1-7:32 (2011)

3. Aguilera, M.K.: A pleasant stroll through the land of infinitely many creatures. SIGACT News 35(2), 36-59 (2004)

4. Aspnes, J.: Notes on theory of distributed systems. http://www.cs.yale.edu/homes/aspnes/classes/465/notes.pdf accessed: 2020-08-08

5. Aspnes, J., Attiya, H., Censor-Hillel, K.: Polylogarithmic concurrent data structures from monotone circuits. J. ACM 59(1), 1-24 (2012)

6. Attiya, H., Bar-Noy, A., Dolev, D.: Sharing memory robustly in message-passing systems. J. ACM 42(1), 124-142 (Jan 1995)

7. Attiya, H., Chung, H., Ellen, F., Kumar, S., Welch, J.: Emulating a shared register in an asynchronous system that never stops changing. TPDS 30(3), 544-559 (2018)

8. Attiya, H., Fouren, A., Gafni, E.: An adaptive collect algorithm with applications. Dist. Comp. 15(2), 87-96 (2002)

9. Attiya, H., Herlihy, M., Rachman, O.: Atomic snapshots using lattice agreement. Dist. Comp. 8(3), 121-132 (1995)

10. Baldoni, R., Bonomi, S., Kermarrec, A.M., Raynal, M.: Implementing a register in a dynamic distributed system. In: ICDCS. pp. 639-647 (2009)

11. Baldoni, R., Bonomi, S., Raynal, M.: Implementing a regular register in an eventually synchronous distributed system prone to continuous churn. TPDS 23(1), 102-109 (2012) 
12. Baldoni, R., Bonomi, S., Raynal, M.: Implementing set objects in dynamic distributed systems. Journal of Computer and System Sciences 82(5), 654- 689 (2016)

13. Castañeda, A., Rajsbaum, S., Raynal, M.: Unifying concurrent objects and distributed tasks: Interval-linearizability. J. ACM 65(6), 1-42 (2018)

14. Chordia, S., Rajamani, S., Rajan, K., Ramalingam, G., Vaswani, K.: Asynchronous resilient linearizability. In: DISC. pp. 164-178 (2013)

15. Delporte-Gallet, C., Fauconnier, H., Rajsbaum, S., Raynal, M.: Implementing snapshot objects on top of crash-prone asynchronous message-passing systems. TPDS 29(9), 2033-2045 (2018)

16. Faleiro, J.M., Rajamani, S., Rajan, K., Ramalingam, G., Vaswani, K.: Generalized lattice agreement. In: PODC. pp. 125-134 (2012)

17. Gafni, E., Malkhi, D.: Elastic configuration maintenance via a parsimonious speculating snapshot solution. In: DISC. pp. 140-153 (2015)

18. Gafni, E., Merritt, M., Taubenfeld, G.: The concurrency hierarchy, and algorithms for unbounded concurrency. In: PODC. pp. 161-169 (2001)

19. Gilbert, S., Lynch, N.A., Shvartsman, A.A.: Rambo: A robust, reconfigurable atomic memory service for dynamic networks. Dist. Comp. 23(4), 225-272 (2010)

20. Herlihy, M.P., Wing, J.M.: Linearizability: A correctness condition for concurrent objects. Trans. Prog. Lang. Sys. 12(3), 463-492 (Jul 1990)

21. Jehl, L., Vitenberg, R., Meling, H.: Smartmerge: A new approach to reconfiguration for atomic storage. In: DISC. pp. 154-169 (2015)

22. Kuznetsov, P., Rieutord, T., Tucci-Piergiovanni, S.: Reconfigurable lattice agreement and applications. In: OPODIS. pp. 31:1-31:17 (2019)

23. Lamport, L.: On interprocess communication. part I: basic formalism. Dist. Comp. 1(2), 77-85 (1986). https://doi.org/10.1007/BF01786227. https://doi.org/10.1007/BF01786227

24. Merritt, M., Taubenfeld, G.: Computing with infinitely many processes. In: DISC. pp. 164178 (2000)

25. Spiegelman, A., Keidar, I.: Dynamic atomic snapshots. In: OPODIS (2016)

26. Zheng, X., Garg, V., Kaippallimalil, J.: Linearizable replicated state machines with lattice agreement. In: OPODIS (2019)

27. Zheng, X., Hu, C., Garg, V.: Lattice agreement in message passing systems. In: DISC (2018) 